\title{
Biochemical Evaluation of Antitumor Activity of Vitamin B17 Alone or in Combination with Platinum Based Drugs Against Ehrlich Ascites Carcinoma in Female Rats
}

Said A. Barakat*, Saleh S. Yousef**, Ibrahim A. Ibrahim**, Yasmina K. Mahmoud**, Marwa A. El-Beltagy**

*Veterinarian; **Biochemistry department, Faculty of Veterinary Medicine, Suez Canal University

*Corresponding author: Email: ashraf.biochemistry@gmail.com Tel.: +201140000974

\section{Abstract}

Chemotherapeutic agents are associated with many side effects. Consequently, much research is interested in the discovery of natural phytochemical compounds that can be used in the prevention and/or treatment of cancer. Ehrlich ascites carcinoma (EAC) model was used to indicate the effectiveness of some chemotherapy and plant sources against cancer due to its similarity with human tumors. The present study was undertaken to investigate antitumor and antioxidant effects of vitamin B17 beside platinum-based drugs in EAC-bearing female rats. Animals were randomly distributed into seven groups $(n=7)$ as follows: Group A, negative control. Group B, positive control that was injected by EAC cells as a cancer model. Group C, EAC-bearing rats were treated with cisplatin. Group D, rats with EAC and were treated by a single dose of oxaliplatin. Group E, rats with EAC and were treated with vitamin B17 (VB17). Group F, rats with EAC and were treated with cisplatin plus vitamin B17. Group G, EAC-bearing rats that were treated by singledose oxaliplatin plus VB17. One week after the beginning of treatments, blood samples were collected and tumor markers (AFP, CEA, CA19-9, TPA, and LDH), as well as antioxidants biomarkers (SOD, CAT, GSH, and MDA), were measured. Liver and kidney functions were evaluated. Besides, histopathological examination was performed to evaluate antitumor activity and side effects of used drugs on hepatic and renal tissues. Results showed that administration of VB17 alone or in combination with cisplatin or oxaliplatin led to a decrease of tumor markers together with enhancement of antioxidant indicators compared with EACbearing rats. Statistical analysis showed a significant 
$(\mathrm{P}<0.05)$ increase in the activities of ALT, AST, ALP accompanied by an increase in the serum levels of creatinine, BUN, and total bilirubin in cisplatin and oxaliplatin treated groups, thus confirming their toxic effects on hepatocytes and renal cells. These findings were supported by histopathological alterations in these groups. VB17 treated groups showed improvement in the studied parameters. From the current study, it could be concluded that vitamin B17 possesses anticancer and antioxidant activities that justify its traditional use, and its potential hepatoprotective effect and kidney ameliorative role.

Keywords: Ehrlich ascites carcinoma, Vitamin B17, Cisplatin, Oxaloplatin, Tumour markers, Antioxidants.

\section{Introduction}

Many plants and phytochemicals exhibit valuable antioxidant activities, which have a significant role in the treatment and prevention of cancer (Abd Eldaim et al., 2019b; Elmasry et al., 2018; Oyouni et al., 2018). Vitamin B17 (VB17), also termed amygdalin and laetrile, is a cyanogenic diglucoside, a type of carbohydrate that is mostly naturally found in the kernel of fruits such as apricot, bitter almond, macadamias, and peach. Many researchers reported that VB17 has numerous medicinal activities including antitussive, antiasthmatic, antiatherogenic, anticancer, anti-inflammation, and anti-ulcer potentials beside its ability to inhibit fibrosis (Juengel et al., 2016; Makarević et al., 2016; Qian et al., 2015). Also, some studies have supported that VB17 can induce apoptotic cell death of several cancer types such as promyelocytic leukemia, cervical, prostatic and hepatic cancer (Chen et al., 2013; Sauer et al., 2015; Zhou et al., 2012).

Various malignancies could be treated by using platinum-based drugs such as cisplatin, oxaliplatin, and carboplatin alone or in combination with other chemotherapeutic agents (Wong and Giandomenico, 1999). Cisplatin [cisdiaminedichloroplatinum (II), CDDP], is the first compound of this group. It blocks DNA replication and RNA transcription which initiates apoptosis process (Wang and Lippard, 2005). Its application is still limited due to the sideeffects associated with its toxicity as well as increasing cisplatin resistant (Brabec and Kasparkova, 2005). 
Oxaliplatin is a third-generation platinum-based drug with 1,2diaminocyclohexane

$(\mathrm{DACH})$ substituting the amine groups of cisplatin (Raymond et al., 2002). It has demonstrated a satisfied safety profile, characterized by low haematotoxicity, fewer inter and intra DNA strands adducts to achieve the same cytotoxicity. The main side effect of this compound is neurotoxicity (Waseem et al., 2017).

Ehrlich ascites carcinoma (EAC) is an undifferentiated, rapidly proliferative, short life span, $100 \%$ malignance spontaneous murine breast adenocarcinoma (Kaleoğlu and Işsi, 1977). It is firstly observed in a female mouse, then extensively studied afterwards using murine models, including mice (Mishra et al., 2018; Sugiura, 1953; Wang, 2013) and rat (Olinici et al., 1975, 1977; Osman et al., 2015; Podoplelov, 1957) to a lesser extent, to investigate tumor pathogenesis and development of anti-tumorigenic agents (Simon et al., 1979) due to its resemblance to human tumors since it is highly sensitive to chemotherapy with rapid growth rates, great transplantable capability and lacks tumorspecific transplantation antigen (TSTA) (Ozaslan et al., 2011). Loewenthal and Jahn (1932) named it as "Ehrlich ascites carcinoma" due to development of the ascites liquid, with carcinoma cells in peritoneum of mouse after intraperitoneal (i.p) injection of cells.

The current study aimed to investigate antioxidant and antitumor activities of VB17 on EAC - bearing female rats alone and in combination with cisplatin or oxaliplatin to ameliorates their side effects on hepatic and renal tissues.

\section{Mate rial and Methods}

Experimental animals :

A total number of 49 female healthy albino rats weighing 120-130 g were used in the current study. They were obtained from the animal laboratory house in Faculty of Veterinary Medicine, Suez Canal University. The animals were housed in plastic cages and maintained under controlled conditions of temperature (23$25^{\circ} \mathrm{C}$ ), relative humidity $(40$ $70 \%$ ) and diurnal environmental (12 h light/dark cycles). All animals had free access to water and standard laboratory rat diet during the experimental period. Rats were acclimatized for seven days before starting the experiment. This study was approved by committee of scientific research and biological ethics for animals used in laboratory experiments in the Faculty of Veterinary 
Medicine, Suez Canal University, Egypt.

\section{Drugs:}

1- Platinol ${ }^{\circledR}$ (cisplatin for injection, USP) is a white to light yellow lyophilized powder. Imported by RAMCO, Manufacturer: Oncotec Pharma Produktion $\mathrm{GmbH}$ - Germany.

2- Oxaliplatin (Eloxatin) is a white to off-white powder or crystals, is slightly soluble in water at $6 \mathrm{mg} / \mathrm{mL}$, very slightly soluble in methanol, and practically insoluble in ethanol and acetone. Imported by Forward Pharma Co. EGY.

3- Vitamin B17 (VB17, Amygdalin) chemical name: [(6-O- $\beta$-D-glucopyranosyl- $\beta$-Dglucopyranosyl) oxy] (phenyl) acetonitrile. Produced by Cytopharma de Mexico, S.A

Tumor cell line (Induction stage):

The parent line of Ehrlich ascites carcinoma cells (EAC cells) was obtained from the National Cancer Institute (NCI), Cairo University, Egypt. EAC cells were collected from donor female Swiss albino mice of 18 - 20 g body weight and suspended in sterile saline $(0.9 \% \mathrm{NaCl})$. A fixed number of viable cells (usually $2.5 \times 10^{6}$ cells/mice) were implanted in the peritoneal cavity of each recipient female rat (Salem $\boldsymbol{e t}$ al., 2011). Every $0.5 \mathrm{ml}$ of EAC was withdrawn by a sterile disposable syringe, diluted with
$4.5 \mathrm{ml}$ of normal saline $(0.9 \%$ $\mathrm{NaCl}$ ). $0.2 \mathrm{ml}$ of diluted EAC was i.p. injected into 42 rats. The tumor cells were allowed to multiply within the peritoneal cavity for 2 weeks (Abouzaid, 2013; Hanafy, 2009).

\section{Experimental Design:}

Animals were randomly divided into seven groups, seven animals each as follow:

Group A
control) served as normal control group. Rats were treated with saline and received standard diet all over the experimental period (3 weeks).

\section{Group B (positive} control) served as EAC control group. Rats were i.p. injected by EAC cells and were not treated all over the experimental period (3 weeks).

Group C (Cisplatin) served as $\mathrm{EAC}+$ cisplatin group. Rats were i.p. injected by EAC cells. Two weeks after the induction phase, the animals were i.p. treated with cisplatin $(12 \mathrm{mg} / \mathrm{kg}$ b.w.) for one week (Miller $\boldsymbol{e t}$ al., 2010).

Group D (Oxaliplatin) served as EAC + oxaliplatin group. Rats were i.p. injected by EAC cells. Two weeks after the induction phase, the animals were treated with a single i.p. injection of oxaliplatin (6 $\mathrm{mg} / \mathrm{kg}$ ) (Ling et al., 2008).

Group E (Vitamin B17) served as $\mathrm{EAC}+\mathrm{VB} 17$ group. Rats were i.p. injected by EAC cells. 
Two weeks after the induction phase, the animals were i.p. treated with VB17 $(4 \mathrm{mg} / \mathrm{kg})$ for one week (Minaiyan et al., 2014).

Group F (Cisplatin + Vitamin B 17) served as EAC + cisplatin + VB17 group. Rats were i.p. injected by EAC cells. Two weeks after the induction phase, the animals were i.p. treated cisplatin (12 mg/kg b.w.) and VB17 (4 mg/kg) for one week.

Group G (Oxaliplatin + Vitamin B 17) served as EAC + oxaliplatin + VB17 group. Rats were i.p. injected by EAC cells. Two weeks after the induction phase, the animals were treated with single i.p. injection of oxaliplatin $(6 \mathrm{mg} / \mathrm{kg})$ and VB17 (4 mg/kg) for one week.

Blood and tissue samples collection:

At the end of the experiment (one week after starting treatments), blood samples were collected from overnight fasted rats from retro orbital venous plexus using micro-hematocrit tubes under the effect of light ether anesthesia. Blood was divided into two tubes; EDTA and plain centrifuge tubes for determination of hematological and biochemical parameters, respectively. Clear serum samples were separated and stored at $-20^{\circ} \mathrm{C}$ till time of analysis. Liver, kidney and spleen were collected and fixed in $10 \%$ formalin for histopathological examinations. Evaluation of hematological parameters:

Hematological parameters were determined by automated hematology system analyzer using whole blood. The assessed parameters include total and differential white blood cells count (WBC), red blood cells count (RBC), hemoglobin ( $\mathrm{Hb})$, hematocrit (HCT), mean cell volume (MCV), mean corpuscular hemoglobin $(\mathrm{MCH})$, mean corpuscular hemoglobin concentration (MCHC) and platelets.

Evaluation of biochemical parameters:

- Determination of tissue antioxidants

Catalase (CAT) (Sigma-Aldrich Co., USA) and superoxide dismutase (SOD) activities, reduced Glutathione (GSH) and malondialdehyde (MDA) were calorimetrically measured using methodology described by (Aebi, 1984; Fossati et al., 1980), (Nishikimi et al., 1972), (Beutler and Gelbart, 1985) and (Satoh, 1978).

- Determination of tumor markers:

Tumor markers, including alpha-fetoprotein (Mcintire et al., 1975; Tatarinov, 1964), carcinoembryonic antigen (CEA) (Thomson et al., 1969; Zamcheck and Martin, 1981), carbohydrate antigen 19-9 
(Koprowski et al., 1981), tissue polypeptide antigen (TPA) (Björklund and Björklund, 1957) (LDH), and lactate dehydrogenase (LDH) (Lorentz et al., 1993), were estimated using kits manufacturer protocol.

- Determination of liver and kidne y functions

Serum activities of alanine amino transferase (ALT), aspartate amino transferase (AST) and alkaline phosphatase (ALP) (Human, Germany) were assayed using the method of (Schumann and Klauke, 2003) and (Moss and Henderson, 1999), respectively. Serum total proteins, albumin, creatinine and blood urea nitrogen (BUN) levels were assayed using methodology described by $\boldsymbol{E l}$ Moghazy et al. (2014), Moustafa et al. (2014), Jaffé (1886) and Chaney and Marbach (1962), respectively. His topathological Evaluation: Organs were processed by standard methods to prepare slides of hepatic, renal and spleen tissues by hematoxylin and eosin (H\&E) staining (ElSayyad et al., 2009; Ray et al., 1981). Then, slides were viewed under light microscope.

Statis tical analysis :

All data were subjected to statistical analysis by using computer programs, SPSS version 18 for analysis of data and Duncan's multiple range test for determination of LSD. Comparison were carried by means using analysis of variance "F test" (ANOVA) where appropriate statistical significance was calculated using least significant difference "LSD". The level of statistical significance was taken as $\mathrm{P}<$ 0.05 .

\section{Results}

\section{Macroscopic observations}

After inoculation of rats with EAC cells, rats exhibited marked enlargement of abdomen with formation of ascitic fluid (Figure 1).

\section{parameters}

Results showed significant $(\mathrm{P}<0.05)$ decrease in $\mathrm{RBCs}$ count, Hb level, PCV, MCV, $\mathrm{MCH}, \mathrm{MCHC}$ as well as PLT count in EAC bearing rats (group B) with significant $(\mathrm{P}<0.05)$ increase in WBCs count when compared with the control group (group A). On the other hand, treating EACbearing rats with either VB17 alone (group $\mathrm{E}$ ) or in combination with oxaliplatin (group G) resulted in a significant $\quad(\mathrm{P}<0.05)$ improvement in previous parameters when compared with other treated groups (groups C, $\mathrm{D}$ and F) (Table 1).

Effect of cisplatin, oxaloplatin and VB17 on antioxidant parameters: 
The effect of cisplatin, oxaloplatin and VB17 on antioxidant status (SOD, CAT, GSH and MDA) of EAC bearing rats are shown in Table (2). Antioxidant enzymes activities (SOD and CAT) as well as GSH level were significantly $(\mathrm{P}<0.05)$ reduced in EAC-induced rats (Groups B, $\mathrm{C}$ and D) compared to the control group (Group A) whereas MDA level was significantly $(\mathrm{P}<0.05)$ elevated. However, treating rats with VB17 either alone or in combination with cisplatin or oxaloplatin improved antioxidant status of rats.

Effect of cisplatin, oxaloplatin and VB17 on tumor biomarkers

Table (3) showed EAC bearing rats (group $\mathrm{B}$ ) exhibited significant $(\mathrm{P}<0.05)$ elevation in serum levels of alphafetoprotein (AFP), carcinoembryonic antigen (CEA), cancer antigen 19-9 (CA 19-9), tissue polypeptide antigen (TPA) and lactate dehydrogenase (LDH) activity as compared with normal rats (group A). On the other hand, treatment of rats with cisplatin (group C), oxaloplatin (group D), VB17 (group E), or their combinations (groups $F$ and $G$ ) resulted in significant $(\mathrm{P}<0.05)$ reduction in serum tumor markers. Moreover, the best results were observed in groups $\mathrm{E}$ and $\mathrm{G}$.

Effect of cisplatin, oxaloplatin and VB17 on liver functions

As shown in Table (4), EAC bearing rats (group $\mathrm{B}, \mathrm{C}$ and $\mathrm{D}$ ) demonstrated significant $(\mathrm{P}<0.05)$ rise in activity of hepatic enzymes (ALT, AST and ALP) in serum meanwhile serum albumin and total bilirubin levels were significantly $(\mathrm{P}<0.05)$ decreased when compared with normal rats (group A). In contrast, treatment of rats VB17 alone or coincided with oxaloplatin (group E, G) resulted in significant $\quad(\mathrm{P}<0.05)$ improvement in hepatic markers.

Effect of Cisplatin, Oxaloplatin and VB17 on kidney function

Results shown in Table (5) demonstrated that rats of group (B, C and F) developed significant $(\mathrm{P}<0.05)$ elevation in serum creatinine and BUN levels as compared with the control rats (group A). While rats of groups (D, E and $G$ ) showed a significant reduction in serum creatinine and BUN levels.

Effect of cisplatin, oxaloplatin and VB17 on hepatic, splenic and renal tissues:

Liver sections of rat bearing Ehrlich ascites carcinoma (Group B) showed various histopathological alternations 
including vacuolization of hepatocellular cytoplasm, sporadic cell necrosis of individual hepatocytes with deeply pyknotic nuclei, congestion of central vein. EAC bearing rats treated with cisplatin (Group C) showed congested portal tract vessel and hydropic degeneration of hepatocytes while treated with oxaliplatin (Group D) showed uniform hepatocytes, with congested central vein. EAC bearing rats and treated with VB17 (Group E) showed uniform hepatocytes with no signs of injury. EAC bearing rats and treated with VB17 (Group E) showed uniform hepatocytes with no signs of injury. EAC bearing rats and treated with cisplatin plus VB17 (Group F) showed hydropic degeneration of hepatocytes, patent sinusoids with congested portal vessel. EAC bearing rats and treated with oxaliplatin plus VB17 (Group G) showed uniform hepatocytes, with mildly congested sinusoids (Figure 2).

While spleen sections of rats bearing EAC (Group B) showed marked expansion of red bulb due to congestion and small uniform lymphoid follicles. In EAC bearing rats and rats treated with cisplatin (Group C) or with oxaliplatin (Group D) spleen sections showed expansion of red bulb due to congestion and atrophic lymphoid follicles. EAC bearing rats and treated with VB17 (Group E) showed weak expansion of red bulb due to slight congestion of lymphoid follicles. EAC bearing rats treated with cisplatin plus VB17 (Group F) or oxaliplatin plus VB17 (Group G) showed expansion of red bulb due to congestion atrophic lymphoid follicles (Figure 3).

Kidney sections of rat bearing EAC (Group B) showed enlarged glomeruli, mesangial expansion and endo-capillary proliferation. Renal tubules show increased evidence of acute tubular injury. In EAC bearing rats treated with cisplatin (Group C) as well as those treated with oxaliplatin (Group D) renal tubules showed evidence of acute tubular injury. EAC bearing rats and treated with VB17 (Group E) showed mild enlarged glomeruli with tubules in addition to evidence of mild acute tubular injury. In EAC bearing rats and treated with cisplatin plus VB17 (Group F) or oxaliplatin plus VB17 (Group G), glomeruli became enlarged with mesangial expansion and endo-capillary proliferation. Tubules showed increased evidence of acute tubular injury (Figure 4). 


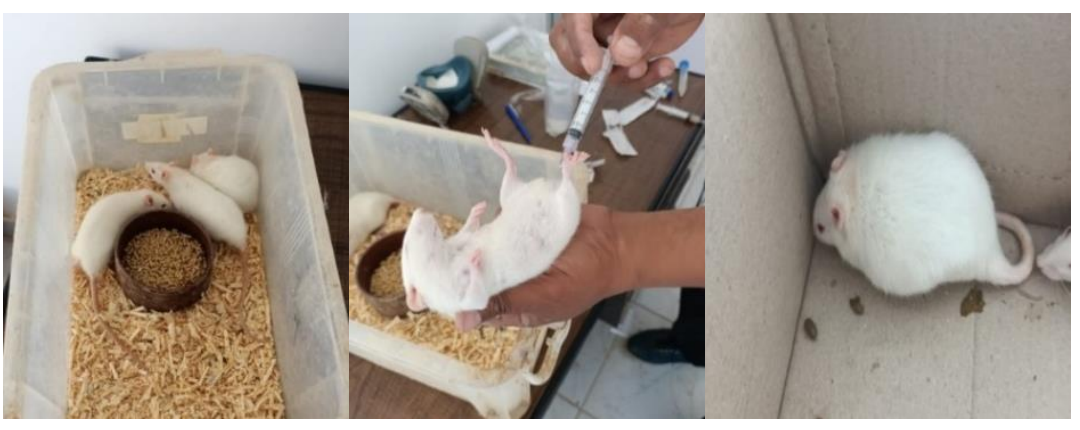

Before induction of During induction of 10 days after induction of EAC

Figure 1: Stages of development of tumor ascitic fluid in rats Effect of cisplatin, oxaloplatin and VB17 on hematological

Table (1): Hematological Parameters in different treated groups

\begin{tabular}{|c|c|c|c|c|c|c|c|}
\hline Groups & $\begin{array}{c}\text { Group } \\
\text { A }\end{array}$ & $\begin{array}{c}\text { Group } \\
\text { B }\end{array}$ & $\begin{array}{l}\text { Group } \\
\text { C }\end{array}$ & $\begin{array}{l}\text { Group } \\
\text { D }\end{array}$ & $\begin{array}{c}\text { Group } \\
\text { E }\end{array}$ & $\begin{array}{c}\text { Group } \\
\text { F }\end{array}$ & $\begin{array}{c}\text { Group } \\
\text { G }\end{array}$ \\
\hline $\begin{array}{c}\text { RBCs } \\
\text { (million/mm) }\end{array}$ & $\begin{array}{c}6.53^{\mathrm{a}} \pm \\
0.13\end{array}$ & $\begin{array}{c}5.23^{\mathrm{d}} \pm \\
0.19\end{array}$ & $\begin{array}{c}5.41^{\mathrm{cd}} \pm \\
0.29\end{array}$ & $\begin{array}{c}5.79^{\mathrm{bc}} \pm \\
0.10\end{array}$ & $\begin{array}{c}6.07^{\mathrm{b}} \pm \\
0.07\end{array}$ & $\begin{array}{c}5.85^{\mathrm{bc}} \pm \\
0.12\end{array}$ & $\begin{array}{c}6.62^{\mathrm{a}} \pm \\
0.10\end{array}$ \\
\hline $\begin{array}{c}\mathrm{Hb} \\
(\mathrm{g} / \mathrm{dl})\end{array}$ & $\begin{array}{c}14.24^{\mathrm{a}} \pm \\
0.49\end{array}$ & $\begin{array}{c}10.50^{\mathrm{d}} \pm \\
0.41\end{array}$ & $\begin{array}{c}10.73^{\mathrm{d}} \pm \\
0.41\end{array}$ & $\begin{array}{c}11.44^{\mathrm{cd}} \pm \\
0.36\end{array}$ & $\begin{array}{c}13.21^{\mathrm{ab}} \pm \\
0.53\end{array}$ & $\begin{array}{c}10.93^{\mathrm{d}} \pm \\
0.22\end{array}$ & $\begin{array}{c}12.57^{\mathrm{bc}} \pm \\
0.38\end{array}$ \\
\hline $\begin{array}{c}\text { PCV } \\
(\text { HCT) }(\mathrm{L} / \mathrm{L})\end{array}$ & $\begin{array}{l}0.44^{\mathrm{a}} \pm \\
0.005\end{array}$ & $\begin{array}{c}0.39^{\mathrm{d}} \pm \\
0.004\end{array}$ & $\begin{array}{c}0.40^{c} \pm \\
0.004\end{array}$ & $\begin{array}{c}0.41^{b c} \pm \\
0.003\end{array}$ & $\begin{array}{l}0.43^{\mathrm{a}} \pm \\
0.003\end{array}$ & $\begin{array}{l}0.40^{\mathrm{c}} \pm \\
0.005\end{array}$ & $\begin{array}{l}0.42^{b} \pm \\
0.005\end{array}$ \\
\hline $\begin{array}{c}\mathrm{MCV} \\
\text { (fl) }\end{array}$ & $\begin{array}{c}57.00^{\mathrm{a}} \pm \\
2.16\end{array}$ & $\begin{array}{c}43.86^{\mathrm{d}} \pm \\
1.32\end{array}$ & $\begin{array}{c}45.29^{\mathrm{cd}} \pm \\
0.52\end{array}$ & $\begin{array}{c}47.71^{\mathrm{bc}} \pm \\
0.52\end{array}$ & $\begin{array}{c}54.71^{\mathrm{a}} \pm \\
1.46\end{array}$ & $\begin{array}{l}47.29^{\mathrm{bcd}} \\
\pm 0.52\end{array}$ & $\begin{array}{c}50.43^{\mathrm{b}} \pm \\
0.81\end{array}$ \\
\hline $\begin{array}{c}\text { MCH } \\
\text { (pg) }\end{array}$ & $\begin{array}{c}21.14^{\mathrm{a}} \pm \\
0.63\end{array}$ & $\begin{array}{c}16.86^{\mathrm{d}} \pm \\
0.34\end{array}$ & $\begin{array}{c}18.71^{\mathrm{c}} \pm \\
0.52\end{array}$ & $\begin{array}{c}19.57^{\mathrm{abc}} \\
\pm 0.48\end{array}$ & $\begin{array}{c}21.14^{\mathrm{a}} \pm \\
0.63\end{array}$ & $\begin{array}{c}19.29^{\mathrm{bc}} \pm \\
0.52\end{array}$ & $\begin{array}{c}20.71^{\mathrm{ab}} \pm \\
0.52\end{array}$ \\
\hline $\begin{array}{c}\mathrm{MCHC} \\
(\mathrm{g} / \mathrm{L})\end{array}$ & $\begin{array}{c}331.43^{\mathrm{a}} \\
\pm 9.86\end{array}$ & $\begin{array}{c}274.29^{\mathrm{d}} \\
\pm 4.81\end{array}$ & $\begin{array}{c}278.57^{\mathrm{d}} \\
\pm 3.40\end{array}$ & $\begin{array}{c}295.71^{\mathrm{c}} \\
\pm 4.81\end{array}$ & $\begin{array}{c}317.14^{\mathrm{ab}} \\
\pm 5.22\end{array}$ & $\begin{array}{c}288.57^{\text {cd }} \\
\pm 3.40\end{array}$ & $\begin{array}{c}311.43^{\mathrm{b}} \\
\pm 3.40\end{array}$ \\
\hline $\begin{array}{l}\text { PLT } \\
\left(10^{9} / \text { L }\right)\end{array}$ & $\begin{array}{l}832.14^{\mathrm{a}} \\
\pm 26.14 \\
\end{array}$ & $\begin{array}{l}702.86^{\mathrm{b}} \\
\pm 16.43 \\
\end{array}$ & $\begin{array}{r}584.29^{c} \\
\pm 14.94 \\
\end{array}$ & $\begin{array}{l}384.29^{\mathrm{e}} \\
\pm 20.22 \\
\end{array}$ & $\begin{array}{l}795.71^{\mathrm{a}} \\
\pm 31.31 \\
\end{array}$ & $\begin{array}{l}654.29^{\mathrm{b}} \\
\pm 16.31 \\
\end{array}$ & $\begin{array}{l}507.14^{\mathrm{d}} \\
\pm 22.65 \\
\end{array}$ \\
\hline $\begin{array}{l}\text { WBCs } \\
\left(10^{9} / \mathrm{L}\right)\end{array}$ & $\begin{array}{c}11.10^{\mathrm{d}} \pm \\
0.39\end{array}$ & $\begin{array}{c}17.00^{\mathrm{a}} \pm \\
0.52 \\
\end{array}$ & $\begin{array}{c}15.31^{\mathrm{b}} \pm \\
0.68\end{array}$ & $\begin{array}{c}13.80^{\mathrm{bc}} \pm \\
0.50\end{array}$ & $\begin{array}{c}11.86^{\mathrm{d}} \pm \\
0.33\end{array}$ & $\begin{array}{c}13.96^{\mathrm{bc}} \pm \\
0.52 \\
\end{array}$ & $\begin{array}{c}12.50^{\text {cd }} \pm \\
0.55\end{array}$ \\
\hline
\end{tabular}

All values were expressed as mean \pm SE. Differences were considered significant at $\mathrm{P}<0.05$.

Table (2) Antioxidant status in different treated groups

\begin{tabular}{|c|c|c|c|c|c|c|c|}
\hline Groups & $\begin{array}{c}\text { Group } \\
\text { A }\end{array}$ & $\begin{array}{c}\text { Group } \\
\text { B }\end{array}$ & $\begin{array}{l}\text { Group } \\
\text { C }\end{array}$ & $\begin{array}{c}\text { Group } \\
\text { D }\end{array}$ & $\begin{array}{l}\text { Group } \\
\mathbf{E}\end{array}$ & $\begin{array}{c}\text { Group } \\
\text { F }\end{array}$ & $\begin{array}{l}\text { Group } \\
\text { G }\end{array}$ \\
\hline $\begin{array}{c}\text { SOD } \\
\left(I U \times 10^{-4}\right) \\
\end{array}$ & $\begin{array}{c}2.29^{\mathrm{a}} \pm \\
0.09 \\
\end{array}$ & $\begin{array}{c}1.14^{\mathrm{b}} \pm \\
0.08\end{array}$ & $\begin{array}{c}1.27^{\mathrm{b}} \pm \\
0.12 \\
\end{array}$ & $\begin{array}{c}1.65^{\mathrm{b}} \pm \\
0.14 \\
\end{array}$ & $\begin{array}{c}2.20^{\mathrm{a}} \pm \\
0.13\end{array}$ & $\begin{array}{c}2.00^{\mathrm{ab}} \pm \\
0.15\end{array}$ & $\begin{array}{c}2.24^{\mathrm{a}} \pm \\
0.15 \\
\end{array}$ \\
\hline $\begin{array}{c}\text { CAT } \\
(\text { IU x 10-2 }) \\
\end{array}$ & $\begin{array}{c}3.54^{\mathrm{a}} \pm \\
0.17 \\
\end{array}$ & $\begin{array}{c}1.47^{\mathrm{b}} \pm \\
0.17\end{array}$ & $\begin{array}{c}1.99^{\mathrm{b}} \pm \\
0.11\end{array}$ & $\begin{array}{c}2.02^{\mathrm{b}} \pm \\
0.10\end{array}$ & $\begin{array}{l}3.40^{\mathrm{a}} \pm \\
0.15\end{array}$ & $\begin{array}{c}2.96^{\mathrm{ab}} \pm \\
0.12\end{array}$ & $\begin{array}{l}3.50^{\mathrm{a}} \pm \\
0.15\end{array}$ \\
\hline $\begin{array}{c}\text { GSH } \\
(\mu \mathrm{mol} / \mathrm{L})\end{array}$ & $\begin{array}{c}5.53^{\mathrm{a}} \pm \\
0.16 \\
\end{array}$ & $\begin{array}{c}3.49^{\mathrm{b}} \pm \\
0.12 \\
\end{array}$ & $\begin{array}{c}3.65^{\mathrm{b}} \pm \\
0.12 \\
\end{array}$ & $\begin{array}{c}4.05^{\mathrm{b}} \pm \\
0.14 \\
\end{array}$ & $\begin{array}{c}5.56^{\mathrm{a}} \pm \\
0.15 \\
\end{array}$ & $\begin{array}{c}5.50^{\mathrm{a}} \pm \\
0.16 \\
\end{array}$ & $\begin{array}{c}5.43^{\mathrm{a}} \pm \\
0.17 \\
\end{array}$ \\
\hline $\begin{array}{c}\text { MDA } \\
\left(\mathrm{mM} / \mathbf{L} \times 10^{-}\right)\end{array}$ & $\begin{array}{c}3.01^{\mathrm{b}} \pm \\
0.33\end{array}$ & $\begin{array}{c}5.49^{\mathrm{a}} \pm \\
0.18\end{array}$ & $\begin{array}{c}4.86^{\mathrm{a}} \pm \\
0.11\end{array}$ & $\begin{array}{c}4.25^{\mathrm{b}} \pm 0 \\
.24\end{array}$ & $\begin{array}{l}2.40^{\mathrm{b}} \pm \\
0.15\end{array}$ & $\begin{array}{c}2.94^{\mathrm{b}} \pm \\
0.19\end{array}$ & $\begin{array}{c}2.80^{\mathrm{b}} \pm \\
0.21\end{array}$ \\
\hline
\end{tabular}

All values were expressed as mean \pm SE. Differences were considered significant at $\mathrm{P}<0.05$. 
Table (3) Tumor biomarkers in different treated groups

\begin{tabular}{|c|c|c|c|c|c|c|c|}
\hline Groups & $\begin{array}{c}\text { Group } \\
\text { A }\end{array}$ & $\begin{array}{c}\text { Group } \\
\text { B }\end{array}$ & $\underset{\text { C }}{\text { Group }}$ & $\begin{array}{c}\text { Group } \\
\text { D }\end{array}$ & $\begin{array}{c}\text { Group } \\
\text { E }\end{array}$ & $\underset{\text { F }}{\text { Group }}$ & $\begin{array}{c}\text { Group } \\
\text { G }\end{array}$ \\
\hline $\begin{array}{c}\text { AFB } \\
(\mathrm{ng} / \mathrm{mL})\end{array}$ & $\begin{array}{c}14.43^{1} \pm \\
1.70\end{array}$ & $\begin{array}{c}60.71^{\mathrm{a}} \pm \\
3.41\end{array}$ & $\begin{array}{c}47.43^{\mathrm{b}} \pm \\
2.49\end{array}$ & $\begin{array}{l}40.14^{\text {cd }} \\
\pm 1.70\end{array}$ & $\begin{array}{c}25.29^{\mathrm{e}} \pm \\
1.64\end{array}$ & $\begin{array}{c}42.71^{\mathrm{bc}} \pm \\
2.56\end{array}$ & $\begin{array}{c}35.29^{\mathrm{d}} \pm \\
1.70\end{array}$ \\
\hline $\begin{array}{c}\text { CEA } \\
(\mathrm{ng} / \mathrm{mL})\end{array}$ & $\begin{array}{c}5.71^{\mathrm{e}} \pm \\
0.36\end{array}$ & $\begin{array}{c}11.43^{\mathrm{a}} \pm \\
0.69\end{array}$ & $\begin{array}{c}10.14^{\mathrm{b}} \pm \\
0.34\end{array}$ & $\begin{array}{c}9.14^{\mathrm{bc}} \pm \\
0.34\end{array}$ & $\begin{array}{c}7.71^{\mathrm{d}} \pm \\
0.36\end{array}$ & $\begin{array}{c}9.14^{\mathrm{bc}} \pm \\
0.34\end{array}$ & $\begin{array}{c}8.00^{\mathrm{cd}} \pm \\
0.38\end{array}$ \\
\hline $\begin{array}{c}\text { CA 19-9 } \\
(\mathrm{U} / \mathrm{ml})\end{array}$ & $\begin{array}{c}18.86^{\mathrm{e}} \pm \\
2.09 \\
\end{array}$ & $\begin{array}{c}61.00^{\mathrm{a}} \pm \\
3.48\end{array}$ & $\begin{array}{c}54.57^{\mathrm{ab}} \pm \\
3.23 \\
\end{array}$ & $\begin{array}{c}47.86^{\mathrm{bc}} \pm \\
2.44 \\
\end{array}$ & $\begin{array}{c}32.71^{\mathrm{d}} \pm \\
2.52 \\
\end{array}$ & $\begin{array}{c}48.29^{\mathrm{bc}} \pm \\
2.51\end{array}$ & $\begin{array}{c}41.86^{\mathrm{c}} \pm \\
2.42 \\
\end{array}$ \\
\hline $\begin{array}{c}\text { TPA } \\
(\mathbf{n g} / \mathrm{ml})\end{array}$ & $\begin{array}{c}1.17^{\mathrm{e}} \pm \\
0.13\end{array}$ & $\begin{array}{c}2.29^{\mathrm{a}} \pm \\
0.05\end{array}$ & $\begin{array}{c}2.03^{\mathrm{b}} \pm \\
0.07\end{array}$ & $\begin{array}{c}1.89^{\mathrm{bc}} \pm \\
0.03\end{array}$ & $\begin{array}{c}1.53^{\mathrm{d}} \pm \\
0.10\end{array}$ & $\begin{array}{c}1.81^{\mathrm{bc}} \pm \\
0.08\end{array}$ & $\begin{array}{c}1.69^{\text {cd }} \pm \\
0.07\end{array}$ \\
\hline $\begin{array}{l}\text { LDH } \\
(\mathrm{U} / \mathrm{l})\end{array}$ & $\begin{array}{c}155.00^{c} \\
\pm 6.37\end{array}$ & $\begin{array}{c}238.43^{\mathrm{a}} \\
\pm 5.14\end{array}$ & $\begin{array}{c}226.00^{\mathrm{a}} \\
\pm 5.15\end{array}$ & $\begin{array}{c}212.71^{\mathrm{b}} \\
\pm 4.01\end{array}$ & $\begin{array}{c}199.71^{\mathrm{b}} \\
\pm 3.36\end{array}$ & $\begin{array}{c}211.14^{\mathrm{b}} \\
\pm 3.36\end{array}$ & $\begin{array}{c}201.00^{\mathrm{b}} \\
\pm 3.39\end{array}$ \\
\hline
\end{tabular}

All values were expressed as mean \pm SE. Differences were considered significant at $\mathrm{P}<0.05$.

Table (4) Liver function in different treated groups

\begin{tabular}{|c|c|c|c|c|c|c|c|}
\hline Groups & $\begin{array}{c}\text { Group } \\
\mathbf{A}\end{array}$ & $\begin{array}{c}\text { Group } \\
\text { B }\end{array}$ & $\underset{\text { Croup }}{\text { Group }}$ & $\begin{array}{c}\text { Group } \\
\text { D }\end{array}$ & $\begin{array}{c}\text { Group } \\
\text { E }\end{array}$ & $\underset{\text { Group }}{\text { F }}$ & $\begin{array}{c}\text { Group } \\
\text { G }\end{array}$ \\
\hline $\begin{array}{c}\text { ALT } \\
\text { (IU/L) }\end{array}$ & $\begin{array}{c}27.86^{\mathrm{f}} \pm \\
4.01\end{array}$ & $\begin{array}{c}304.29^{\mathrm{a}} \\
\pm \\
16.16\end{array}$ & $\begin{array}{c}224.29^{\mathrm{b}} \\
\pm \\
8.41\end{array}$ & $\begin{array}{c}175.71^{\mathrm{cd}} \\
\pm \\
8.41\end{array}$ & $\begin{array}{c}124.29^{\mathrm{e}} \\
\pm \\
8.41\end{array}$ & $\begin{array}{c}202.14^{\mathrm{bc}} \\
\pm \\
8.30\end{array}$ & $\begin{array}{c}152.14^{\mathrm{d}} \\
\pm \\
8.30\end{array}$ \\
\hline $\begin{array}{c}\text { AST } \\
(\text { IU/L) }\end{array}$ & $\begin{array}{c}90.71^{\mathrm{e}} \pm \\
13.11^{\mathrm{N}}\end{array}$ & $\begin{array}{c}394.29^{\mathrm{a}} \\
\pm \\
16.88\end{array}$ & $\begin{array}{c}325.71^{\mathrm{b}} \\
\pm \\
8.41\end{array}$ & $\begin{array}{c}271.43^{\mathrm{c}} \\
\pm \\
8.29\end{array}$ & $\begin{array}{c}195.71^{\mathrm{d}} \\
\pm \\
16.35\end{array}$ & $\begin{array}{c}252.86^{\mathrm{c}} \\
\pm \\
8.30\end{array}$ & $\begin{array}{c}206.43^{\mathrm{d}} \\
\pm \\
12.14\end{array}$ \\
\hline $\begin{array}{c}\text { ALP } \\
\text { (IU/L) }\end{array}$ & $\begin{array}{c}63.57^{\mathrm{d}} \pm \\
10.45\end{array}$ & $\begin{array}{c}187.14^{\mathrm{a}} \\
\pm \\
19.70\end{array}$ & $\begin{array}{c}167.86^{\mathrm{ab}} \\
\pm \\
11.54\end{array}$ & $\begin{array}{c}140.00^{\mathrm{bc}} \\
\pm \\
9.82\end{array}$ & $\begin{array}{c}122.14^{\mathrm{c}} \\
\pm \\
8.72\end{array}$ & $\begin{array}{c}142.86^{\mathrm{bc}} \\
\pm \\
10.11\end{array}$ & $\begin{array}{c}130.00^{\mathrm{c}} \\
\pm \\
9.70\end{array}$ \\
\hline $\begin{array}{c}\text { Albumin } \\
(\mathrm{g} / \mathrm{dL})\end{array}$ & $\begin{array}{c}3.87^{\mathrm{a}} \pm \\
0.27\end{array}$ & $\begin{array}{c}2.59^{c} \pm \\
0.25\end{array}$ & $\begin{array}{c}2.57^{\mathrm{c}} \pm \\
0.16\end{array}$ & $\begin{array}{c}2.89^{b c} \pm \\
0.16\end{array}$ & $\begin{array}{c}3.37^{\mathrm{ab}} \pm \\
0.16\end{array}$ & $\begin{array}{c}2.83^{\mathrm{bc}} \pm \\
0.15\end{array}$ & $\begin{array}{c}3.13^{\mathrm{bc}} \pm \\
0.15\end{array}$ \\
\hline $\begin{array}{c}\text { Total } \\
\text { bilirubin } \\
(\mathrm{mg} / \mathrm{dL})\end{array}$ & $\begin{array}{c}0.97^{\mathrm{d}} \pm \\
0.07\end{array}$ & $\begin{array}{l}1.84^{\mathrm{a}} \pm \\
0.08\end{array}$ & $\begin{array}{c}1.73^{\mathrm{ab}} \pm \\
0.06\end{array}$ & $\begin{array}{c}1.54^{\mathrm{b}} \pm \\
0.06\end{array}$ & $\begin{array}{c}1.16^{\mathrm{cd}} \pm \\
0.10\end{array}$ & $\begin{array}{c}1.59^{\mathrm{b}} \pm \\
0.07\end{array}$ & $\begin{array}{l}1.33^{\mathrm{c}} \pm \\
0.05\end{array}$ \\
\hline
\end{tabular}

All values were expressed as mean \pm SE. Differences were considered significant at $\mathrm{P}<0.05$.

Table (5) Kidney function in different treated groups

\begin{tabular}{|c|c|c|c|c|c|c|c|}
\hline $\begin{array}{c}\text { Croups } \\
\text { Parameter }\end{array}$ & $\begin{array}{c}\text { Group } \\
\text { A }\end{array}$ & $\begin{array}{c}\text { Group } \\
\text { B }\end{array}$ & $\begin{array}{c}\text { Group } \\
\text { C }\end{array}$ & $\begin{array}{c}\text { Group } \\
\text { D }\end{array}$ & $\begin{array}{c}\text { Group } \\
\text { E }\end{array}$ & $\begin{array}{c}\text { Group } \\
\text { F }\end{array}$ & $\begin{array}{c}\text { Group } \\
\text { G }\end{array}$ \\
\hline $\begin{array}{c}\text { BUN } \\
(\mathbf{m g} / \mathbf{d L})\end{array}$ & $\begin{array}{c}18.00^{\mathrm{d}} \pm \\
1.13\end{array}$ & $\begin{array}{c}21.71^{\mathrm{bc}} \pm \\
1.36\end{array}$ & $\begin{array}{c}26.86^{\mathrm{a}} \pm \\
0.40\end{array}$ & $\begin{array}{c}19.57^{\mathrm{cd}} \pm \\
1.41\end{array}$ & $\begin{array}{c}18.29^{\mathrm{d}} \pm \\
1.15\end{array}$ & $\begin{array}{c}23.00^{\mathrm{b}} \pm \\
0.38\end{array}$ & $\begin{array}{c}18.57^{\mathrm{cd}} \pm \\
1.11\end{array}$ \\
\hline $\begin{array}{c}\text { Serum } \\
\text { creatinine } \\
\text { (mg/dL) }\end{array}$ & $\begin{array}{c}0.59^{\mathrm{d}} \pm \\
0.07\end{array}$ & $\begin{array}{c}1.36^{\mathrm{a}} \pm \\
0.15\end{array}$ & $\begin{array}{c}1.27^{\mathrm{ab}} \pm \\
0.16\end{array}$ & $\begin{array}{c}1.00^{\mathrm{bc}} \pm \\
0.07\end{array}$ & $\begin{array}{c}0.61^{\mathrm{d}} \pm \\
0.07\end{array}$ & $\begin{array}{c}1.19^{\mathrm{ab}} \pm \\
0.06\end{array}$ & $\begin{array}{c}0.73^{\mathrm{cd}} \pm \\
0.07\end{array}$ \\
\hline
\end{tabular}

All values were expressed as mean \pm SE. Differences were considered significant at $\mathrm{P}<0.05$. 

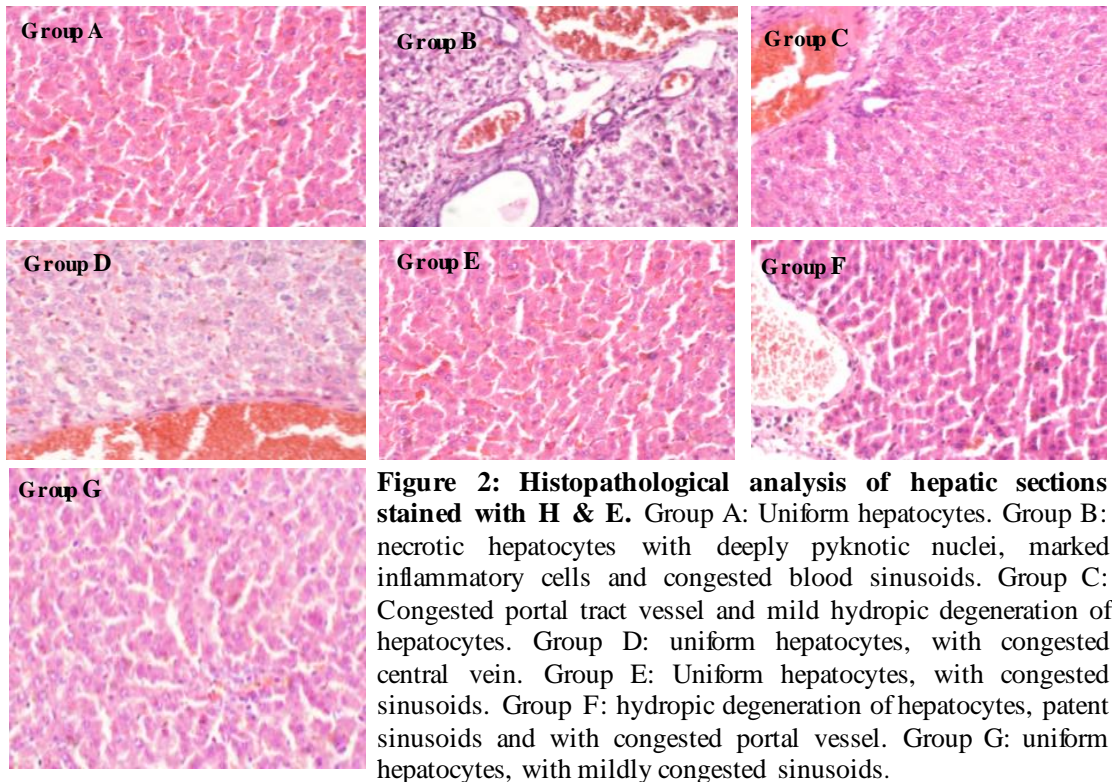

Figure 2: Histopathological analysis of hepatic sections stained with $\mathbf{H} \&$ E. Group A: Uniform hepatocytes. Group B: necrotic hepatocytes with deeply pyknotic nuclei, marked inflammatory cells and congested blood sinusoids. Group C: Congested portal tract vessel and mild hydropic degeneration of hepatocytes. Group D: uniform hepatocytes, with congested central vein. Group E: Uniform hepatocytes, with congested sinusoids. Group F: hydropic degeneration of hepatocytes, patent sinusoids and with congested portal vessel. Group G: uniform hepatocytes, with mildly congested sinusoids.
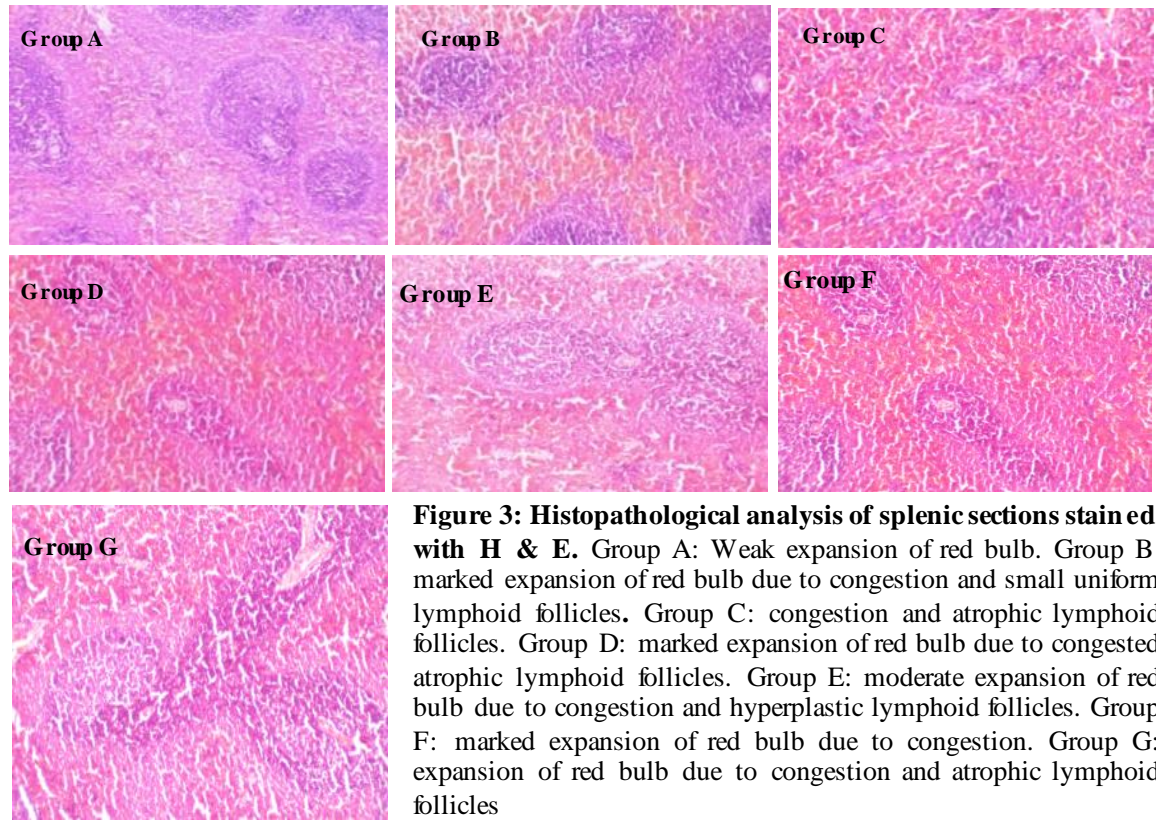

Figure 3: Histopathological analysis of splenic sections stain ed with H \& E. Group A: Weak expansion of red bulb. Group B: marked expansion of red bulb due to congestion and small uniform lymphoid follicles. Group C: congestion and atrophic lymphoid follicles. Group D: marked expansion of red bulb due to congested atrophic lymphoid follicles. Group E: moderate expansion of red bulb due to congestion and hyperplastic lymphoid follicles. Group F: marked expansion of red bulb due to congestion. Group G: expansion of red bulb due to congestion and atrophic lymphoid follicles 


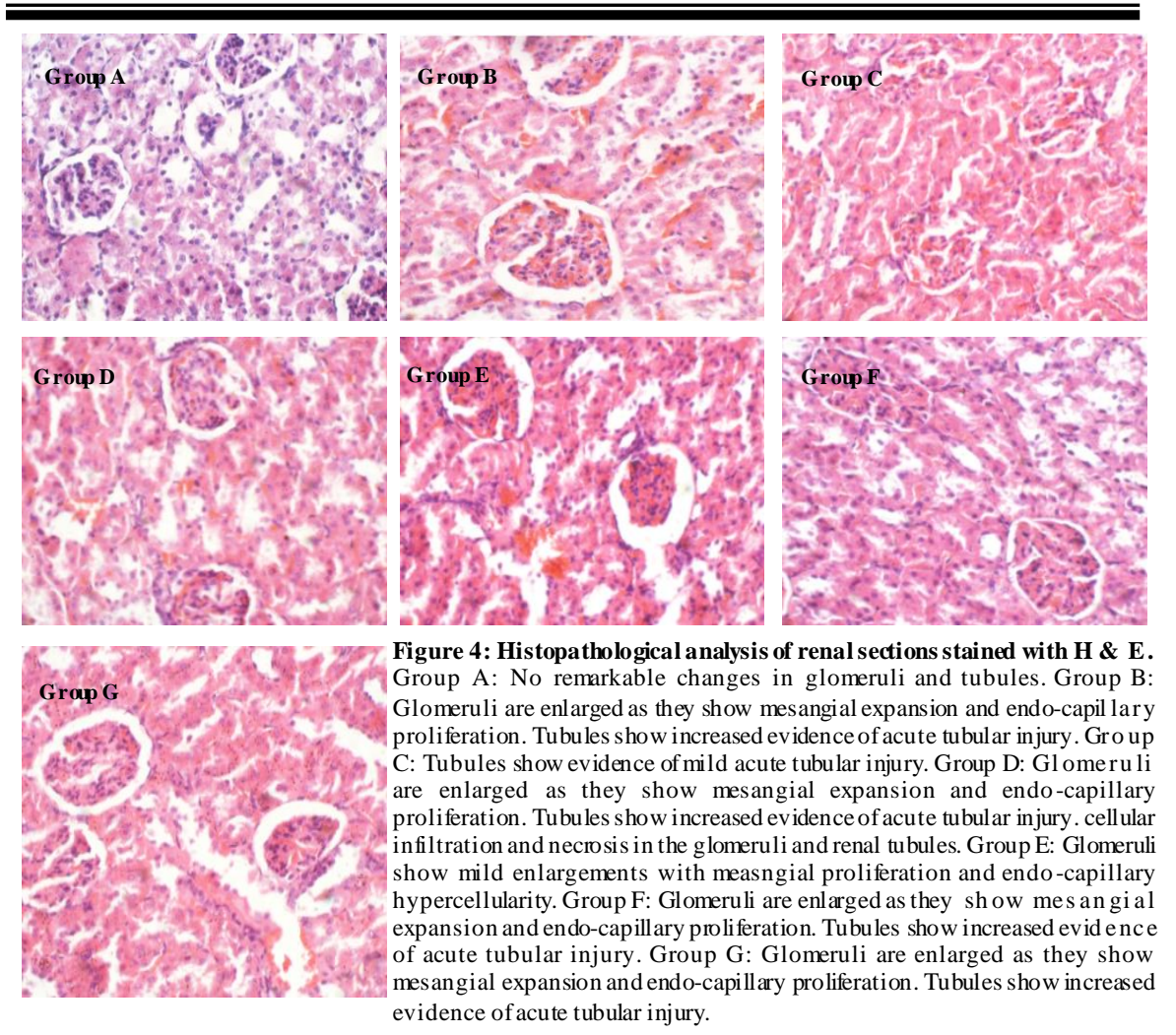

\section{Discussion}

Ehrlich carcinoma has been used to investigate the antitumor effects of numerous natural and synthetic chemical substances (David et al., 2019). Consequently, the present study aimed to examine the possible defensive, curative properties, hepatic and renal ameliorative role of vitamin B17 against Ehrlich ascites carcinoma in comparison with antitumor activities of cisplatin and oxaliplatin as anticancer drugs. In accordance, we evaluate the deleterious changes in tumor markers and antioxidant status, liver and kidney functions in EAC - bearing female rats. The current study revealed that i.p injection of Ehrlich cells induced a rapid increase in ascitic fluid volume in EAC bearing rats in untreated group (Group B) as shown in Figure 1 . This come in agreement with Hackensellner and Hermanek (1958); Osman et al. (2015); Stroud et al. (1957) and Alotaibi et al. (2021); Funasaka et al. (2002); Hashem et al. (2020) who studied Ehrlich ascites tumors in rats and mice, respectively. Our results revealed that Ehrlich tumor-induced alterations in hematological parameters including decrease in RBCs count, $\mathrm{Hb}, \mathrm{PCV}$, and gradual 
increase in PLT and WBCs, as well induced changes in MCV, $\mathrm{MCH}$, and $\mathrm{MCHC}$ levels. These results are consistent with those reported by Agrawal et al. (2011); Mutar et al. (2019); Perveen et al. (2012). This could be explained by the suppressive influence of EAC on bone marrow erythropoiesis. However, observed the granulocytic leucocytosis may be due to development of stress in response to increased fluid ascites cells or acute inflammatory response $(\boldsymbol{A L}$ Mashhadani et al., 2018).

Data of current study demonstrated marked $(\mathrm{P}<0.05)$ reduction in SOD and CAT activities and GSH content whereas elevation of MDA content in EAC bearing rats as compared with that of normal control rats. In accordance with our results, Haldar et al. (2010) recorded depletion of SOD activity and GSH level coincided with elevated MDA level in tumor-bearing animals.

Our findings indicated that EAC induces significant $\quad(\mathrm{P}<0.05)$ increase in tumor markers including AFP, CEA, CA 19-9, TPA and LDH. Alphafetoprotein is a commonly used tumor marker for diagnosis of hepatocellular carcinoma (Tangkijvanich et al., 2000) as well as estimation of tumor size (Qin and Tang, 2002). Similar results were also obtained by
Perkins et al. (2003) who reported that, an increase in CEA and CA19-9 levels is associated with adenocarcinoma, especially colorectal cancer. Also, elevated TPA levels can also be detected in some benign events such as liver failure, renal failure, gestation, generalized infection, and diabetes mellitus (Tramonti et al., 2000). Further, Samudrala et al. (2015) observed that intraperitoneal inoculation of EAC cells is associated with a significant $(\mathrm{P}<0.05)$ increase in serum activity of LDH which could be attributed to hepatocellular damage induced by EAC.

Ehrlich carcinoma caused abnormalities in liver functions which is indicated by increased activities of serum enzymes ALT, AST and ALP beside marked elevation in total bilirubin level and diminished albumin level compared to normal control group. The current results come in agree with results obtained by Haldar et al. (2010). Our findings indicated that the observed impairment of liver functions could be a direct consequence of both disruption of cellular redox balance together with cancer development. It has been reported that increased lipid peroxidation and inhibition of GSH content, catalase and SOD activity led to liver and kidney 
dysfunction (Borges et al., 2006). Moreover, these data are supported by histopathological examination of hepatic sections which revealed increased number of necrotic hepatocytes with deeply pyknotic nuclei, congestion associated with brown pigment deposition and thickening of wall on the central vein. Similar results were reported by Ali et al. (2015) and Badr et al. (2011).

Besides, Ehrlich tumor has been shown to induce kidney injury and negatively influence renal function. This is evidenced by increased levels of BUN and creatinine. Similarly, Habib et al. (2010); Khanam et al. (2010) demonstrated that EAC led to elevation of serum urea, creatinine potassium and chloride ions whereas decreased sodium ions. Histopathological examination of kidney sections showed marked degeneration in glomeruli and some parts of the urinary tubules in kidney sections in EAC-bearing group. These results are in harmony with Abd Eldaim et al. (2019a); Badr et al. (2011); El-Wahab and Fouda (2009); Medhat et al. (2017); Salem et al. (2011) who recorded histopathological alteration in renal tissue which varied from cellular infiltration to degenerated renal tubules and atrophied glomeruli following induction of EAC.
The adverse effects of cisplatin on hematological parameters were demonstrated as significantly $(\mathrm{P}<0.05)$ diminished RBCs and platelets counts with subsequent reduction in the values of $\mathrm{Hb} \%$, $\mathrm{MCV}, \mathrm{MCH}, \mathrm{MCHC}$ and $\mathrm{PCV}$ together with elevation of WBCs. Previous studies proposed that there is a reasonable relationship between cisplatin treatment and occurrence of anemia. This could be clarified via various mechanisms including increment of RBCs osmotic fragility or deterioration of cells of bone marrow. Consequently, cisplatin intoxication could result in anemia due to either disruption of erythropoiesis, prohibition of hematopoietic tissues activity or hastened RBCs breakdown due to alteration of membrane permeability of RBCs (Yuan et al., 2014). Furthermore, Marković et al. (2011) showed that apart from the diminished RBC count, prolonged cisplatin application could trigger a decline in platelets count and an elevation in WBCs count of rats. The reduction in platelets count could arise from inhibition of bone marrow activity by cisplatin or might be due to reduced synthesis or elevated consumption of platelets or due to the excess platelets aggregation (Sirag, 
2009). In this consequence, Olas et al. (2005) stated that cisplatin induces oxidative stress (OS) in human platelets and lymphocytes, which might negatively affect their life span, and subsequently trigger apoptosis, thus decreasing these cells number in the blood.

epatotoxicity induced by cisplatin is detected by the alterations of the histological, biochemical and molecular parameters (Attyah and Ismail, 2012; El-Sayyad et al., 2009; Karadeniz et al., 2011). In the current experiment, the cisplatin injected rats showed elevation of activities of serum enzymes of ALT, AST, ALP together with depression of serum level of albumin when compared with negative control group. As the elevation in the serum activity of liver cytoplasmic enzyme, ALT indicates necrotic lesions in the hepatic cells. On the other hand, the decline in serum albumin level indicates that there was a deterioration in both synthetic and execratory activities of the liver ( $\boldsymbol{E}$ Sharaky et al., 2009). Histopathological examination of hepatic tissue in cisplatin treated groups are in line with the previously observed parameters and histological alterations in hepatic sections of this group. The current results come in agreement with Abdelmeguid et al. (2010) who manifested marked alterations in hepatic tissue following cisplatin treatment.

Our study revealed marked increment of serum AFP level in cisplatin treated group compared to the control. Numerous studies have revealed that serum AFP concentration elevates in response to exposure to hepatotoxic or hepatocarcinogenic agents (Abass et al., 2018). However, in the current study, cisplatin treated rats exhibited a decrement in the levels of tumor markers compared to untreated EAC rats. This could be explained by the anticancer capabilities of cisplatin. The current results are in accordance with results of $\boldsymbol{A}$ bdel-Hamid $\boldsymbol{e t}$ al. (2011).

It has been documented that cisplatin-induced hepatotoxicity and nephrotoxicity are related to reactive oxygen species (ROS). The elevated ROS attacks the membrane lipids generating the lipid peroxides, which are manifested by increased MDA. The increased MDA level depleted vitamin $\mathrm{E}$, vitamin $\mathrm{C}$, and GSH (Abdel-Raheem et al., 2009). Data from the current study revealed that depletion of GSH, SOD and CAT levels after cisplatin administration might be in response to cisplatin induced oxidative stress. The observed elevation of hepatic enzymes together with increased 
total bilirubin confirm cisplatin hepatotoxicity. This was augmented by pathological alteration in hepatocyte architecture in this group. In addition, it has been documented that cisplatin induce a renal tubular damage which is manifested by impaired reabsorption which is characterized by reduced glomerular filtration rate, increased serum creatinine and blood urea concentrations (Hanigan and Devarajan, 2003; Miller et al., 2010). In this study, histopathological and biochemical evaluation of cisplatin-induced structural alterations and degree of functional alterations in the kidneys were performed in order to determine cisplatininduced nephrotoxicity. Also, histopathological evaluation of renal section of cisplatin treated rats (Group C) augmented cisplatin-induced nephrotoxic effect.

Results of the current study showed development of anemia following oxaliplatin treatment. Evaluation of blood from oxaliplatin-treated rats indicated decreased WBC count and macrocytic anemia. Oxaliplatin is well known to be deleterious to RBC (Fazio et al., 2015) and could directly interact with $\mathrm{Hb}$ (Mandal et al., 2004). Later on, oxaliplatin interaction with hemoglobin has been documented (Potenzieri et al., 2020). Oxaliplatin induced thrombocytopenia occurs mainly due to suppression of bone marrow in a manner similar to other compounds related to the platinum family (Curtis et al., 2006).

The current study revealed that oxaliplatin could induce OS. This is obvious in context of the significantly increased levels of MDA, as well as the markedly decreased antioxidant defense mechanisms (CAT, SOD, and GSH). These results are in harmony with those reported by Robinson et al. (2013). Alterations in hepatic SOD and GSH levels may be explained as a consequence to protein oxidation induced by oxaliplatin treatment in liver mitochondria which results in elevation of superoxide production which in turn impair liver defense mechanism against OS induced by oxaliplatin (Fernandez et al., 2005).

Chan et al. (2009) demonstrated the predictive and monitoring roles of the AFP in hepatic ascites carcinoma (HAC) in rats receiving oxaliplatin-based chemotherapy with extrahepatic spreading. They found significant elevation in AFP after i.p oxaliplatin administration. They suggested that integration of AFP response into the criteria which evaluate treatment. Such consequence 
should be considered in both clinical practice and trials of novel chemotherapeutic agents for treating hepatic carcinoma. In this study, a reported increase in the levels of CEA \& CA 19-9 after treating rats with oxaloplatin. Oxaliplatin has been confirmed to induce inflammatory response, which sounds to be one of the mechanisms of its toxicity. Moreover, elevated CEA level has been correlated with development of inflammation and this was found to agree with the results obtained by Kwon et al. (2018).

It has been reported that oxaliplatin causes elevation of ALT and AST activities and level of total bilirubin (Gurzu et al., 2013). The increased production of bilirubin could be due to the suppressed bilirubin metabolism or obstruction of the bile ducts. Hepatotoxicity induced by oxaliplatin is mainly manifested as hepatic steatosis beside sinus injury (RubbiaBrandt et al., 2010). Furthermore, El Chediak et al. (2018) has manifested that Oxaliplatin hepatotoxicity is most likely associated with splenomegaly in addition to triggering systemic inflammation and elevation of OS.

Unlike cisplatin, oxaliplatin, has been documented to exerts minimal impact on humans and rat kidney (Launay-Vacher et al., 2008; Simpson et al., 2003). This is supported in our study by the suppressed urea and creatinine levels in oxaliplatin treated group more than control positive and cisplatin treated groups. In a study of the pharmacokinetic and toxicodynamic relationships of platinum compounds, it has been documented that the cause for the different tendency of cisplatin and oxaliplatin to development nephrotoxicity is mainly pharmacokinetic in origin besides the total clearance of oxaliplatin was the greatest among the latest platinum compounds (Hanada et al., 2010).

According to our result, hematological parameters were almost restored back to normal range when EAC rats were treated with VB17. Also, VB17 was found to improve WBCs and PLT count efficiently. The $\mathrm{MCV}, \mathrm{MCH}$, and $\mathrm{MCHC}$ levels were observed to be in the normal range. According to $\boldsymbol{A L}$ Mashhadani et al. (2018) VB17 treatment depleted the elevations in AFP levels. This was also emphasized by the findings of Aldubayan et al. (2019); Bruce et al. (2008); Choi and Kakar (2017) who documented that the elevation of serum AFP level might indicate hepatic inflammatory activity and could be 
accompanied by elevation of AST, ALT, and ALP enzyme activities.

Additionally,

Makarević et al. (2016) reported that VB17 possesses a different mechanism through its acquisition on the primary tumor cell's integrin structure which suggests the ability of vitamin B17 to delay the EAC growth in rats.

The current study demonstrated that VB17 was efficiently controlled antioxidant defense system via elevating the levels of catalase, GSH and SOD, whereas decreasing the levels of MDA which indicates antioxidant properties and freeradical scavenging capability of vitamin B17 extract. From these results, we can suggest that vitamin B17 have powerful effects for the treatment of liver cancer when compared with control positive groups.

It was evidenced that VB17 has hepatic ameliorative potential against EAC, which is emphasized by decline of serum AST, ALT, and ALP and elevations of albumin and reduction in total bilirubin levels. The regulation of AST and ALT activities by VB17 supports the possibility that hepatoprotective effect of VB17 occurs through enhancement of antioxidant defense system as together with its scavenging and antioxidant potentials $(\boldsymbol{A L}$ Mashhadani et al., 2018). Badr et al. (2011) reported VB17 could effectively alleviate liver damage through maintaining plasma membrane integrity thereby repressing leakage of enzyme via membranes and consequently exhibit hepatoprotective activity. This might be a reason for restoration of activities of enzymes after administration of Vitamin B17.

Vitamin B17 also exerts renal ameliorative capacity against EAC induced renal injury in female rats. This is obvious by reduced levels serum urea and creatinine in this group. Furthermore, our results were consistent with Salem et al. (2011) who reported that the EAC results in renal dysfunction and elevates serum urea and creatinine levels. These effects were reversed following VB17 treatment. Moreover, Juengel et al. (2016) reported that VB17 could inhibit the kidney cell carcinoma development in rats.

\section{Conclusion}

The present study demonstrated reduced levels of tumor markers, liver enzymes, BUN, creatinine and MDA and enhanced antioxidant indicators (CAT, GSH and SOD) in the EAC group treated with vitamin B17. This indicates the antineoplastic and antioxidant properties exerted by vitamin B17 and suggests that vitamin B17 can be used as a reliable 
and novel therapy for EAC or used in combination with chemotherapeutic agents to overcome their side effects.

\section{References}

Abass, S.A.; Abdel-Hamid, N.M.; Abouzed, T.K.; ElShishtawy, M.M. (2018): Chemosensitizing effect of Alpinia officinarum rhizome extract in cisplatin-treated rats with hepatocellular carcinoma. Biomed Pharmacother, 101, 710-718.

Abd Eldaim, M.A.; Tousson, E.; El Sayed, I.E.T.; Abd ElAleim, A.E.H.; Elsharkawy, H.N. (2019a): Grape seeds proanthocyanidin extract ameliorates Ehrlich solid tumor induced renal tissue and DNA damage in mice. Biomed Pharmacother, 115, 108908.

Abd Eldaim, M.A.; Tousson, E.; El Sayed, I.E.T.; Awd, W.M. (2019b): Ameliorative effects of Saussurea lappa root aqueous extract against Ethephon-induced reproductive toxicity in male rats. Environ Toxicol, 34, (2): 150-159.

Abdel-Hamid, N.M.; Nazmy, M.H.; Mahmoud, A.W.; Fawzy, M.A.; Youssof, M. (2011): A survey on herbal management of hepatocellular carcinoma. World J Hepatol, 3, (7): 175-183.
Abdel-Raheem, I.T.; AbdelGhany, A.A.; Mohamed, G.A. (2009): Protective effect of quercetin against gentamicininduced nephrotoxicity in rats. Biol Pharm Bull, 32, (1): 61-67.

Abde lmeguid, N.E.; Chmaisse, H.N.; Abou Zeinab, N.S. (2010): Silymarin ameliorates cisplatin-induced hepatotoxicity in rats: histopathological and ultrastructural studies. Pak J Biol Sci, 13, (10): 463-479.

Abouzaid, O. (2013): Biochemical effect of some antioxidant on metabolic changes in experimentally induced tumor in female mice. Innovations in pharmaceuticals and pharmacotherapy, 1, 16-22.

Aebi, H. (1984): Catalase in vitro. Methods Enzymol, 105, 121-126.

Agrawal, S.S.; Saras wati, S.; Mathur, R.; Pandey, M. (2011): Antitumor properties of Boswellic acid against Ehrlich ascites cells bearing mouse. Food Chem Toxicol, 49, (9): 1924-1934.

AL-Mashhadani, $\quad$ F.A.; QadirSalihi, A.; Wasman, N. (2018): Effect of apricot kernel on some hematological, histological and biochemical parameters in $\mathrm{CCl}$-induced liver injury in rats. Al-Kitab journal for pure sciences, 2, (1): 217-228. 
Aldubayan,

M.A.;

Elgharabawy, R.M.; Ahmed, A.S.; Tousson, E. (2019): Antineoplastic Activity and Curative Role of Avenanthramides against the Growth of Ehrlich Solid Tumors in Mice. Oxid Med Cell Longev, 2019, 5162687.

Ali, D.A.; Badr El-Din, N.K.; Abou-El-magd, R.F. (2015): Antioxidant and hepatoprotective activities of grape seeds and skin against Ehrlich solid tumor induced oxidative stress in mice. Egyptian Journal of Basic and Applied Sciences, 2, (2): 98109.

Alotaibi, B.; Tousson, E.; ElMasry, T.A.; Altwaijry, N.; Saleh, A. (2021): Ehrlich ascites carcinoma as model for studying the cardiac protective effects of curcumin nanoparticles against cardiac damage in female mice. Environmental Toxicology, 36, (1): 105-113.

Attyah, A.; Ismail, S. (2012): Protective effect of ginger extract against cisplatin-induced hepatotoxicity and cardiotoxicity in rats. Iraqi journal of pharmaceutical sciences, 21, (1): 27-33.

Badr, M.O.; Edrees, N.M.; Abdallah, A.A.; El-Deen, N.A.; Neamat-Allah, A.N.; Ismail, H.T. (2011): Anti- tumour effects of Egyptian propolis on Ehrlich ascites carcinoma. Vet Ital, 47, (3): 341-350.

Beutler, E.; Gelbart, T. (1985): Plasma glutathione in health and in patients with malignant disease. $\mathrm{J}$ Lab Clin Med, 105, (5): 581-584.

Björklund, B.; Björklund, V. (1957): Antigenicity of pooled human malignant and normal tissues by cyto-immunological technique: prescence of an insoluble heat-labile tumor antigen. Int Arch Allergy, 10, 153-184.

Borges, L.P.; Nogueira, C.W.; Panatie ri, R.B.; Rocha, J.B.T.; Zeni, G. (2006): Acute liver damage induced by 2nitropropane in rats: effect of diphenyl diselenide on antioxidant defenses. Chem Biol Interact, 160, (2): 99-107.

Brabec, V.; Kasparkova, J. (2005): Modifications of DNA by platinum complexes. Relation to resistance of tumors to platinum antitumor drugs. Drug Resist Updat, 8, (3): 131146.

Bruce, M.G.; Bruden, D.; McMahon, B.J.; Christensen, C.; Homan, C.; Sullivan, D.; Deubner, H.; Williams, J.; Livingston, S.E.; Gretch, D. (2008): Clinical significance of elevated alpha-fetoprotein in Alaskan Native patients with 
chronic hepatitis C. J Viral Hepat, 15, (3): 179-187.

Chan, S.L.; Mo, F.K.; Johnson, P.J.; Hui, E.P.; Ma, B.B.; Ho, W.M.; Lam, K.C.; Chan, A.T.; Mok, T.S.; Yeo, W. (2009): New utility of an old marker: serial alpha-fetoprotein measurement in predicting radiologic response and survival of patients with hepatocellular carcinoma undergoing systemic chemotherapy. J Clin Oncol, 27, (3): 446-452.

Chaney, A.L.; Marbach, E.P. (1962): Modified reagents for determination of urea and ammonia. Clin Chem, 8, 130132.

Chen, Y.; Ma, J.; Wang, F.; Hu, J.; Cui, A.; Wei, C.; Yang, Q.; Li, F. (2013): Amygdalin induces apoptosis in human cervical cancer cell line HeLa cells. Immunopharmacol Immunotoxicol, 35, (1): 43-51.

Choi, W.T.; Kakar, S. (2017): Immunohistochemistry in the Diagnosis of Hepatocellular Carcinoma. Gastroenterol Clin North Am, 46, (2): 311-325.

Curtis, B.R.; Kalisze wski, J.; Marques, M.B.; Saif, M.W.; Nabelle, L.; Blank, J.; McFarland, J.G.; Aster, R.H. (2006): Immune-mediated thrombocytopenia resulting from sensitivity to oxaliplatin. Am J Hematol, 81, (3): $193-$ 198.
David, I.M.B.; de Souza Fernandes, F.; dos Santos Silva Ferreira, J.B.; Lüdtke, D.D.; Martins, D.F.; Bobinski, F.; da Silva, T.B.G.C.; B uffon, L.D.; Kopper, M.B.R.; da Silva, G.S.; Zefe rino, R.C.; Pedrosa, R.C.; Kviecinski, M.R. (2019): Dietary supplementation with procyanidin-rich Pinus pinaster extract is associated with attenuated Ehrlich tumor development in mice. Nutrition Research, 62, 41-50.

El-Moghazy, M.; Zedan, N.; El-Atrsh, A.; El-Gogary; Tousson, E. (2014): The possible effect of diets containing fish oil (omega-3) on hematological, biochemical and histopathogical alterations of rabbit liver and kidney. Biomed \& Preventive Nutrition, 4, (3): 371-377.

El-Sayyad, H.I.; Is mail, M.F.; Shalaby, F.M.; Abou-ElMagd, R.F.; Gaur, R.L.; Fernando, A.; Raj, M.H.G.; Ouhtit, A. (2009): Histopathological effects of cisplatin, doxorubicin and 5flurouracil (5-FU) on the liver of male albino rats. Int $\mathrm{J}$ Biol Sci, 5, (5): 466-473.

El-Sharaky, A.S.; Newairy, A.A.; Kamel, M.A.; Eweda, S.M. (2009): Protective effect of ginger extract against bromobenzene-induced hepatotoxicity in male rats. 
Food Chem Toxicol, 47, (7): 1584-1590.

El-Wahab, S.E.A.; Fouda, F., 2009. Histological and histochemical study on the effect of Ehrlich ascites carcinoma on the liver and kidney of mice and the possible protective role of tetrodotoxin.

El Che diak, A.; Haydar, A.A.; Hakim, A.; Massih, S.A.; Hilal, L.; Mukherji, D.; Temraz, S.; Shamseddine, A. (2018): Increase in spleen volume as a predictor of oxaliplatin toxicity. Ther Clin Risk Manag, 14, 653-657.

Elmasry, T.; Al-Shaalan, N.; Tousson, E.; Elmorshedy, K.; Al-Ghadeer, A. (2018): Star anise extracts modulation of reproductive parameters, fertility potential and DNA fragmentation induced by growth promoter Equigan in rat testes. Brazilian Journal of Pharmaceutical Sciences, 54.

Fazio, A.; Briglia, M.; Faggio, C.; Alzoubi, K.; Lang, F. (2015): Oxaliplatin Induced Suicidal Death of Human Erythrocytes. Cell Physiol Biochem, 37, (6): 2393-2404.

Fernandez, F.G.; Ritter, J.; Goodwin, J.W.; Linehan, D.C.; Hawkins, W.G.; Strasberg, S.M. (2005): Effect of steatohepatitis associated with irinotecan or oxaliplatin pretreatment on resectability of hepatic colorectal metastases. J Am Coll Surg, 200, (6): 845853.

Fossati, P.; Prencipe, L.; Berti, G. (1980): Use of 3,5dichloro-2-

hydroxybenzenesulfonic acid/4aminophenazone chromogenic system in direct enzymic assay of uric acid in serum and urine. Clin Chem, 26, (2): 227-231.

Funasaka, T.; Haga, A.; Raz, A.; Nagase, H. (2002): Tumor autocrine motility factor induces hyperpermeability of endothelial and mesothelial cells leading to accumulation of ascites fluid. Biochem Biophys Res Commun, 293, (1): 192200.

Gurzu, S.; Jung, I.; Comsulea, M.; Kadar, Z.; Azamfirei, L.; Molnar, C. (2013): Lethal cardiotoxicity, steatohepatitis, chronic pancreatitis, and acute enteritis induced by capecitabine and oxaliplatin in a 36-year-old woman. Diagnostic Pathology, 8, (1): 150.

Habib, M.; Aziz, M.; Karim, M. (2010): Inhibition of Ehrlich's ascites carcinoma by ethyl acetate extract from the flower of Calotropis gigantea L. in mice. Journal of applied biomedicine, 8, (1): 47-54.

Hackensellner, H.A.; Hermanek, P. (1958): Influenceability of subcutaneous transplantation of Ehrlich mouse 
ascites tumor in rats. Oncologia, 11, (3-4): 199-217.

Haldar, P.K.; Kar, B.; Bala, A.; Bhattacharya, S.; Mazumder, U.K. (2010): Antitumor activity of Sansevieria roxburghiana rhizome against Ehrlich ascites carcinoma in mice. Pharmaceutical Biology, 48, (12): 1337-1343.

Hanada, K.; Suda, M.; Kanai, N.; Ogata, H. (2010): Pharmacokinetics and toxicodynamics of oxaliplatin in rats: application of a toxicity factor to explain differences in the nephrotoxicity and myelosuppression induced by oxaliplatin and the other platinum antitumor derivatives. Pharm Res, 27, (9): 1893-1899.

Hanafy, Z.E. (2009): Ginger extract antimutagens as cancer chemopreventive agent against Ehrlich Ascites Carcinoma. Academic journal of cancer research, 2, 61-67.

Hanigan, M.H.; De varajan, P. (2003):

Cisplatin nephrotoxicity: molecular mechanisms. Cancer Ther, 1, 47-61.

Hashem, M.A.; Shoeeb, S.B.A.; Abd-Elhakim, Y.M.; Mohamed, W.A.M. (2020): The antitumor activity of Arthrospira platensis and/or cisplatin in a murine model of Ehrlich ascites carcinoma with hematinic and hepato-renal protective action. Journal of Functional Foods, 66, 103831.

Jaffé, M. (1886): Ueber den Niederschlag, welchen Pikrinsäure in normalem Harn erzeugt und über eine neue Reaction des Kreatinins.

Juengel, E.; Thomas, A.; Rutz, J.; Makarevic, J.; Tsaur, I.; Nelson, K.; Haferkamp, A.; Blaheta, R.A. (2016): Amygdalin inhibits the growth of renal cell carcinoma cells in vitro. Int J Mol Med, 37, (2): 526-532.

Kaleoğlu, Ö.; İş li, N. (1977): Ehrlich-Lettre Asit Tümörü. Tip Fakültesi Mecmuas1, 40, 978984.

Karadeniz, A.; Simsek, N.; Karakus, E.; Yildirim, S.; Kara, A.; Can, I.; Kisa, F.; Emre, H.; Turkeli, M. (2011): Royal jelly modulates oxidative stress and apoptosis in liver and kidneys of rats treated with cisplatin. Oxid Med Cell Longev, 2011, 981793.

Khanam, J.A.; Islam, M.F.; Jesmin, M.; Ali, M.M. (2010): Antineoplastic activity of acetone semicarbazone (ASC) against Ehrlich ascites carcinoma (EAC) bearing mice. Journal of the national science foundation of Sri Lanka, 38, (4): 225-231. 
Koprows ki, H.; Herlyn, M.; Steplewski, Z.; Sears, H.F. (1981): Specific antigen in serum of patients with colon carcinoma. Science, 212, (4490): 53-55.

Kwon, Y.J.; Lee, H.S.; Shim, J.Y.; Lee, Y.J. (2018): Serum carcinoembryonic antigen is positively associated with leukocyte count in Korean adults. J Clin Lab Anal, 32, (3).

Launay-Vacher, V.; Rey, J.B.; Isnard-Bagnis, C.; Deray, G.; Daouphars, M. (2008): Prevention of cisplatin nephrotoxicity: state of the art and recommendations from the European Society of Clinical Pharmacy Special Interest Group on Cancer Care. Cancer Chemother Pharmacol, 61, (6): 903-909.

Ling, B.; Coudoré, F.; Decalonne, L.; Eschalier, A.; Authier, N. (2008): Comparative antiallodynic activity of morphine, pregabalin and lidocaine in a rat model of neuropathic pain produced by one oxaliplatin injection. Neuropharmacology, 55, (5): 724-728.

Loewenthal, H.; Jahn, G. (1932): Übertragung-suersuche mit carcinomatöser mauseasciteslussigleit und İhr verhalten gegen physikalische und chemische einwirkungen. $\mathrm{Z}$. Krebsforsch, 37, 439-447.
Lorentz, K.; Klauke, R.; Schmidt, E. (1993): Recommendation for the determination of the catalytic concentration of lactate dehydrogenase at 37 degrees $\mathrm{C}$. Standardization Committee of the German Society for Clinical Chemistry, Enzyme Working Group of the German Society for Clinical Chemistry. Eur J Clin Chem Clin Biochem, 31, (12): 897-899.

Makarević, J.; Tsaur, I.; Juengel, E.; Borgmann, H.; Nelson, K.; Thomas, C.; Bartsch, G.; Haferkamp, A.; Blaheta, R.A. (2016): Amygdalin delays cell cycle progression and blocks growth of prostate cancer cells in vitro. Life Sci, 147, 137-142.

Mandal, R.; Kalke, R.; Li, X.F. (2004): Interaction of oxaliplatin, cisplatin, and carboplatin with hemoglobin and the resulting release of a heme group. Chem Res Toxicol, 17, (10): 1391-1397.

Marković, S.D.; Žižić, J.B.; Đačić, D.S.; Obradović, A.D.; Ćurčić, M.G.; Cvetković, D.M.; Đorđević, N.Z.; Ognjanović, B.I.; Štajn, A. (2011): Alteration of oxidative stress parameters in red blood cells of rats after chronic in vivo treatment with cisplatin and selenium. Archives of Biological Sciences, 63, (4): 991-999. 
Mcintire, K.R.; Waidmann, T.A.; Moertel, C.G.; Go, V.L.W. (1975): Serum alphafetoprotein in patients with neoplasms of the gastrointestinal tract. Cancer Res., 35, (991-996).

Medhat, D.; Husse in, J.; ElNaggar, M.E.; Attia, M.F.; Anwar, M.; Latif, Y.A.; Booles, H.F.; Morsy, S.; Farrag, A.R.; Khalil, W.K.B.; El-Khayat, Z. (2017): Effect of Au-dextran NPs as anti-tumor agent against EAC and solid tumor in mice by biochemical evaluations and histopathological investigations. Biomed Pharmacother, 91, 1006-1016.

Miller, R.P.; Tadagavadi, R.K.; Ramesh, G.; Reeves, W.B. (2010): Mechanisms of Cisplatin nephrotoxicity. Toxins (Basel), 2, (11): 2490-2518.

Minaiyan, M.; Ghannadi, A.; Asadi, M.; Etemad, M.; Mahzouni, P. (2014): Antiinflammatory effect of Prunus armeniaca L. (Apricot) extracts ameliorates TNBS-induced ulcerative colitis in rats. Res Pharm Sci, 9, (4): 225-231.

Mishra, S.; Tamta, A.K.; Sarikhani, M.; Desingu, P.A.; Kizkekra, S.M.; Pandit, A.S.; Kumar, S.; Khan, D.; Raghavan, S.C.; Sundaresan, N.R. (2018): Subcutaneous Ehrlich Ascites Carcinoma mice model for studying cancerinduced cardiomyopathy. Sci Rep, 8, (1): 5599.

Moss, D.; Henderson, A.R. (1999): Clinical Enzymology, In: Burtis, C.A. and Ashwood, E.R., Eds., Tietz Textbook of Clinical Chemistry, 3rd Edition, Saunders, Philadephia, pp. 617677.

Moustafa, A.H.; Ali, E.M.; Moselhey, S.S.; Tousson, E.; El-Said, K.S. (2014): Effect of coriander on thioacetamideinduced hepatotoxicity in rats. Toxicol Ind Health, 30, (7): 621-629.

Mutar, T.F.; Gazia, M.A.; Salem, S.B.; Hammed, E.H.; Tousson, E. (2019): Ehrlich ascites carcinoma bearing mice as model of human hepatocellular carcinoma. Asian journal of research and reports in hepatology, 1, (1): 1-9.

Nishikimi, M.; Appaji Rao, N.; Yagi, K. (1972): The occurrence of superoxide anion in the reaction of reduced phenazine methosulfate and molecular oxygen. Biochemical and Biophysical Research Communications, 46, (2): 849854.

Olas, B.; Wachowicz, B.; Majsterek, I.; Blasiak, J. (2005): Resveratrol may reduce oxidative stress induced by platinum compounds in human plasma, blood platelets and 
lymphocytes. Anti-cancer drugs, 16, (6): 659-665.

Olinici, C.D.; Rişca, R.; Todoruţiu, C. (1975): Cytogenetic evolution of Ehrlich ascites tumor in rats. A chromosome-banding study. Oncology, 32, (2): 73-81.

Olinici, C.D.; Rişca, R.; Todoruţiu, C. (1977): Chromosomes of lymph node metastases derived from Ehrlich ascites carcinoma cells inoculated into rats. $\mathrm{Z}$ Krebsforsch Klin Onkol Cancer Res Clin Oncol, 90, (3): 281284.

Osman, A.-M.M.; Alqahtani, A.A.; Damanhouri, Z.A.; AlHarthy, S.E.; ElShal, M.F.; Ramadan, W.S.; Kamel, F.; Osman, M.A.M.; Khan, L.M. (2015): Dimethylsulfoxide excerbates cisplatin-induced cytotoxicity in Ehrlich ascites carcinoma cells. Cancer Cell Int, 15, 104-104.

Oyouni, A.A.A.; Saggu, S.; Tousson, E.; Rehman, H. (2018): Immunosuppressant drug tacrolimus induced mitochondrial nephrotoxicity, modified PCNA and Bcl-2 expression attenuated by Ocimum basilicum L. in CD1 mice. Toxicol Rep, 5, 687-694.

Ozaslan, M.; Karagoz, I.D.; Kilic, I.H.; Guldur, M.E. (2011): Ehrlich ascites carcinoma. African journal of biotechnology, 10, (13): 2375 2378.

Perkins, G.L.; Slater, E.D.; Sanders, G.K.; Prichard, J.G. (2003): Serum tumor markers. Am Fam Physician, 68, (6): 1075-1082.

Perveen, R.; Islam, F.; Khanum, J.; Yeasmin, T. (2012): Preventive effect of ethanol extract of Alpinia calcarata Rosc on Ehrlich's ascitic carcinoma cell induced malignant ascites in mice. Asian Pac J Trop Med, 5, (2): 121125.

\section{Podoplelov, II (1957):}

[Prolonged heterogenous transplantation of mouse tumor in rats. I. Studies on biological properties of mouse ascites adenocarcinoma during serial transplantation in rats]. Biull Eksp Biol Med, 43, (6): 63-65.

Potenzieri, A.; Riva, B.; Rigolio, R.; Chiorazzi, A.; Pozzi, E.; Ballarini, E.; Cavaletti, G.; Genazzani, A.A. (2020): Oxaliplatin-induced neuropathy occurs through impairment of haemoglobin proton buffering and is reversed by carbonic anhydrase inhibitors. Pain, 161, (2): 405415.

Qian, L.; Xie, B.; Wang, Y.; Qian, J. (2015): Amygdalinmediated inhibition of nonsmall cell lung cancer cell 
invasion in vitro. Int $\mathrm{J}$ Clin Exp Pathol, 8, (5): 5363-5370.

Qin, L.X.; Tang, Z.Y. (2002): The prognostic significance of clinical and pathological features in hepatocellular carcinoma. World J Gastroenterol, 8, (2): 193-199.

Ray, M.; Guhathakurta, S.; Chowdhury, J.R. (1981): Hematological-changes in experimental-tumors. Indian Journal of Medical Research, 74, (DEC): 896-903.

Raymond, E.; Faivre, S.; Chaney, S.; Woynarowski, J.; Cvitkovic, E. (2002): Cellular and molecular pharmacology of oxaliplatin. Mol Cancer Ther, 1, (3): 227-235.

Robins on, S.M.; Mann, J.; Vasilaki, A.; Mathers, J.; Burt, A.D.; Oakley, F.; White, S.A.; Mann, D.A. (2013): Pathogenesis of FOLFOX induced sinusoidal obstruction syndrome in a murine chemotherapy model. J Hepatol, 59, (2): 318-326.

Rubbia-Brandt, L.; Lauwers, G.Y.; Wang, H.; Majno, P.E.; Tanabe, K.; Zhu, A.X.; B rezault, C.; Soubrane, O.; Abdalla, E.K.; Vauthey, J.N.; Mentha, G.; Terris, B. (2010): Sinusoidal obstruction syndrome and nodular regenerative hyperplasia are frequent oxaliplatin-associated liver lesions and partially prevented by bevacizumab in patients with hepatic colorectal metastasis. Histopathology, 56, (4): 430-439.

Salem, F.S.; Badr, M.O.; Neamat-Allah, A.N. (2011): Biochemical and pathological studies on the effects of levamisole and chlorambucil on Ehrlich ascites carcinomabearing mice. Vet Ital, 47, (1): 89-95.

Samudrala, P.K.; Augustine, B.B.; $\quad$ Kasala, E.R.; Bodduluru, L.N.; Barua, C.; Lahkar, M. (2015): Evaluation of antitumor activity and antioxidant status of Alternanthera brasiliana against Ehrlich ascites carcinoma in Swiss albino mice. Pharmacognosy Res, 7, (1): 6673.

Satoh, K. (1978): Serum lipid peroxide in cerebrovascular disorders determined by a new colorimetric method. Clin Chim Acta, 90, (1): 37-43.

Sauer, H.; Wollny, C.; Oster, I.; Tutdibi, E.; Gortner, L.; Gottschling, S.; Meyer, S. (2015): Severe cyanide poisoning from an alternative medicine treatment with amygdalin and apricot kernels in a 4-year-old child. Wiener Medizinische Wochenschrift, 165, (9): 185-188.

Schumann, G.; Klauke, R. (2003): New IFCC reference 
procedures for the determination of catalytic activity concentrations of five enzymes in serum: preliminary upper reference limits obtained in hospitalized subjects. Clin Chim Acta, 327, (1-2): 69-79.

Simon, P.; Burlingham, W.J.; Conklin, R.; Fondy, T.P. (1979): N-bromoacetyl-beta-Dglucosamine tetra-O-acetate and N-bromoacetyl-beta-Dgalactosamine tetra-O-acetate as chemotherapeutic agents with immunopotentiating effects in Ehrlich ascites tumor-bearing mice. Cancer Res, 39, (10): 3897-3902.

Simpson, D.; Dunn, C.; Curran, M.; Goa, K.L. (2003): Oxaliplatin: a review of its use in combination therapy for advanced metastatic colorectal cancer. Drugs, 63, (19): 21272156.

Sirag, H. (2009): Biochemical and hematological studies for the protective effect of oyster mushroom (Pleurotus ostreatus) against glycerol-induced Acute Renal Failure in rats. Journal of Biological sciences, 9, (7): 746752.

Stroud, A.N.; Brues, A.M.; Chatterley, D.H.; Summers, M. (1957): Serial transplantation of Krebs-2 and Ehrlich ascites tumors to rats. Cancer Res, 17, (11): 11021107.
Sugiura, K. (1953): Effect of various compounds on the Ehrlich ascites carcinoma. Cancer Res, 13, (6): 431-441.

Tangkijvanich, Anukulkarnkusol, Suwangool, P.; Lertmaharit, S.; Hanvivatvong, $\quad 0$.; Kullavanijaya, $\quad$ P.; Poovorawan, Y. (2000): Clinical characteristics and prognosis of hepatocellular carcinoma: analysis based on serum alpha-fetoprotein levels. J Clin Gastroenterol, 31, (4): 302308.

Tatarinov, L.S. (1964): Detection of embryo-specific alpha-globulin in the blood serum of a patient with primary liver cancer. Vopr Med Khim, 10, 90-91.

Thomson, D.M.; Krupey, J.; Freedman, S.O.; Gold, P. (1969): The radioimmunoassay of circulating carcinoembryonic antigen of the human digestive system. Proc Natl Acad Sci U S A, 64, (1): 161-167.

Tramonti, G.; Ferdeghini, M.; Donadio, C.; Norpoth, M.; Annichiarico, C.; Bianchi, R.; Bianchi, C. (2000): Renal function and serum concentration of five tumor markers (TATI, SCC, CYFRA 21-1, TPA, and TPS) in patients without evidence of neoplasia. Cancer Detect Prev, 24, (1): 8690. 
Wang, D.; Lippard, S.J. Yuan, G.; Dai, S.; Yin, Z.; Lu, (2005): Cellular processing of platinum anticancer drugs. Nat Rev Drug Discov, 4, (4): 307320.

Wang, Y. (2013): An experimental study on the antiEhrlich ascites carcinoma effect of purified toad venom extract. Afr J Tradit Complement Altern Med, 10, (6): 547-550.

Waseem, M.; Sahu, U.; Salman, M.; Choudhury, A.; Kar, S.; Tabassum, H.; Parvez, S. (2017): Melatonin pre-treatment mitigates SHSY$5 \mathrm{Y}$ cells against oxaliplatin induced mitochondrial stress and apoptotic cell death. PLoS One, 12, (7): e0180953.

Wong, E.; Giandomenico, C.M. (1999): Current Status of Platinum-Based Antitumor Drugs. Chemical Reviews, 99, (9): 2451-2466.

H.; Jia, R.; Xu, J.; Song, X.; Li, L.; Shu, Y.; Zhao, X. (2014): Toxicological assessment of combined lead and cadmium: acute and subchronic toxicity study in rats. Food and chemical toxicology, 65, 260-268.

Zamcheck, N.; Martin, E.W. (1981):

Sequential

Carcinoembryoic Antigen levels in Pancreatic Cancer: Some Clinical Correlations. Cancer, 1, (47): 1620-1627.

Zhou, C.; Qian, L.; Ma, H.; Yu, X.; Zhang, Y.; Qu, W.; Zhang, X.; Xia, W. (2012): Enhancement of amygdalin activated with $\beta$-D-glucosidase on HepG2 cells proliferation and apoptosis. Carbohydr Polym, 90, (1): 516-523. 


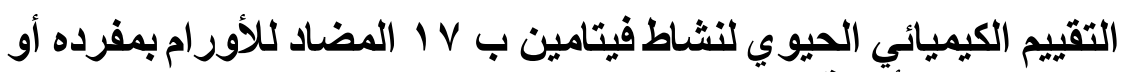
بالاشتر اكمع الأدوية التي تحتوي على البلاتين ضد سرطان استشفقاء إيرليخ

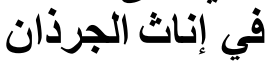

أشرف بركات سيد، شريف يوسف صالح، إبر اهيم عاشور إبر اهيم، ياسمينا كمال محمود، مروة أحمد البلتاجي إبي عائي

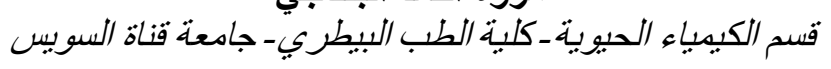

ترتبط أدوية العلاج الكيميائي بالعديد من الآثار الجانبية. وبالتالي، فإن الكثير من الأبحاث

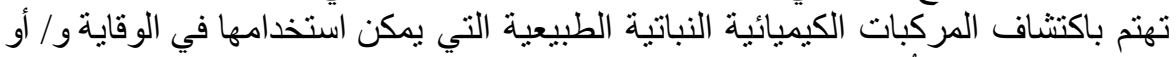

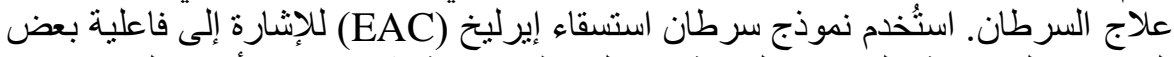

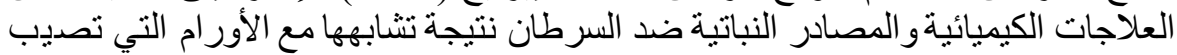

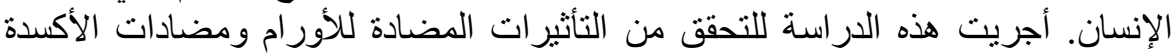

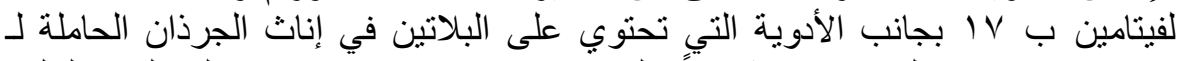
EAC

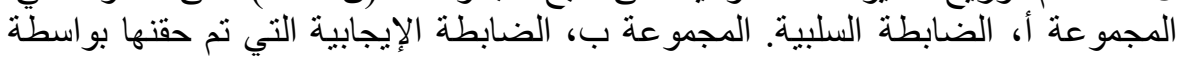

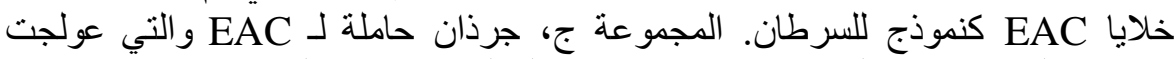

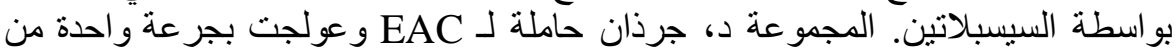

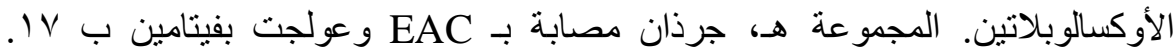

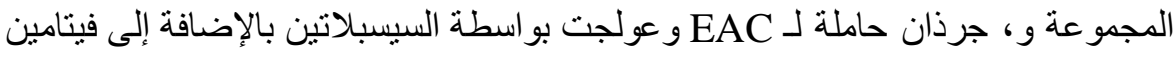

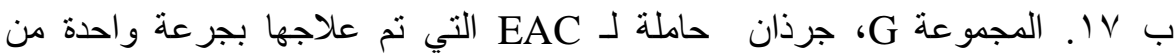

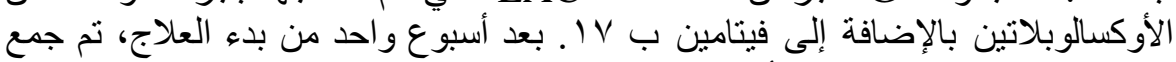

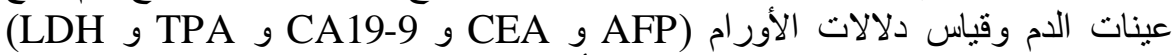
بالإضافة إلى المؤشرات الحيوية لمضادات الأكسدة ( SOD و

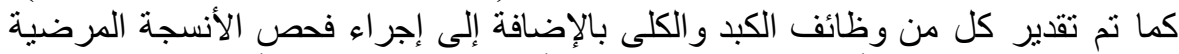

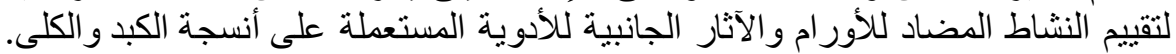

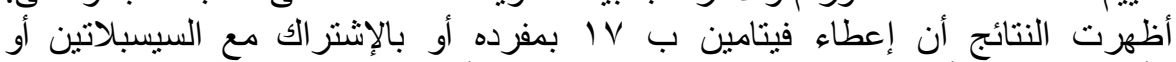

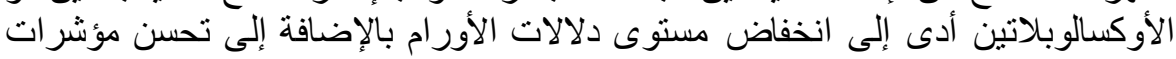

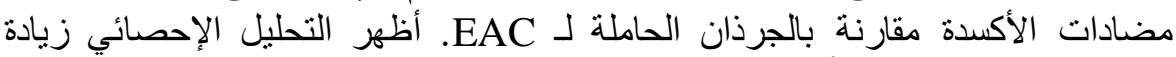

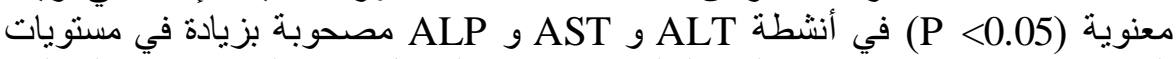

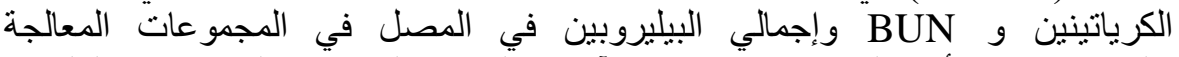

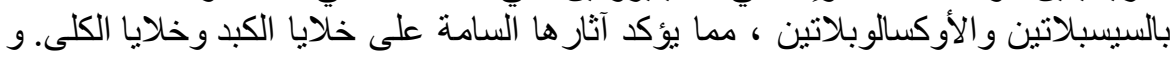

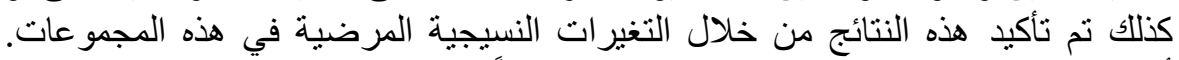

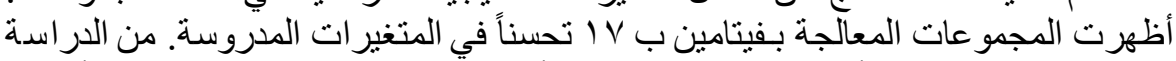

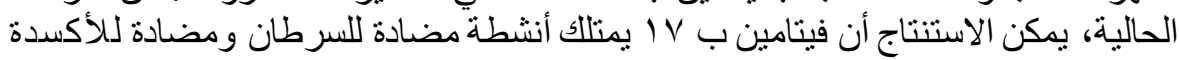
مما يدعم استخدامه المحتمل لحماية الكبد و تحسين وظنئن لائف الكلى. 